Pacific

Journal of

Mathematics

\title{
SPLITTING FIELDS OF G-VARIETIES
}

Zinovy Reichstein AND Boris Youssin 


\title{
SPLITTING FIELDS OF G-VARIETIES
}

\author{
ZinOvy Reichstein AND Boris Youssin
}

Let $G$ be an algebraic group, $X$ a generically free $G$-variety, and $K=k(X)^{G}$. A field extension $L$ of $K$ is called a splitting field of $X$ if the image of the class of $X$ under the natural map $H^{1}(K, G) \mapsto H^{1}(L, G)$ is trivial. If $L / K$ is a (finite) Galois extension then $\operatorname{Gal}(L / K)$ is called a splitting group of $X$.

We prove a lower bound on the size of a splitting field of $X$ in terms of fixed points of nontoral abelian subgroups of $G$. A similar result holds for splitting groups. We give a number of applications, including a new construction of noncrossed product division algebras.

\section{Contents}

1. Introduction 207

2. $G$-varieties 211

3. Two interpretations of $H^{1} \quad 216$

4. Splitting fields 218

5. Examples 221

6. Splitting groups 226

7. Abelian subgroups of $\mathrm{PGL}_{n} \quad 229$

8. Symplectic modules and division algebras 232

9. Constructing a $G$-variety with prescribed stabilizers 237

$\begin{array}{ll}\text { References } & 247\end{array}$

\section{Introduction.}

Let $k$ be an algebraically closed field of characteristic zero, let $K$ be a finitely generated field extension of $k$ and let $G$ is an algebraic group defined over $k$. Recall that elements of the nonabelian cohomology set $H^{1}(K, G)$ can be identified with (birational classes of) generically free $G$-varieties $X$ such that $k(X)^{G}=K$ (see [Po, Section 1.3]). The set $H^{1}(K, G)$ has no group structure in general; however, $H^{1}(K, G)$ is equipped with a marked element, which we shall denote by 1 . This element is represented by the "split" $G$ variety $X \simeq X_{0} \times G$, where $k\left(X_{0}\right)=K$ and $G$ acts by left multiplication on 
the second factor. A field extension $L / K$ is said to be a splitting field for $u \in H^{1}(K, G)$ if $u \mapsto 1$ under the natural map $H^{1}(K, G) \longrightarrow H^{1}(L, G)$.

The nonabelian cohomology set $H^{1}(K, G)$ often allows a different interpretation: Its elements can be identified with certain algebraic objects defined over $K$, e.g, quadratic forms if $G=O_{n}$, central simple algebras if $G=\mathrm{PGL}_{n}$, Cayley algebras, if $G=G_{2}$, etc. These objects may be viewed as "twisted forms" of a single "split" object. In such cases the above notion of a splitting field coincides with the usual one. We will review this interpretation of $H^{1}(K, G)$ in Section 3; see also [Se4, Chapter III], [Se2, Chapter X], [KMRT, Section 29] or [Re, Sections 6-8].

Recall that a subgroup of $G$ is called toral if it lies in a torus in $G$. Our main results on splitting fields are Theorems 1.1 and 1.2.

Theorem 1.1. Let $X$ be a generically free primitive $G$-variety, $K=k(X)^{G}$, and let $L / K$ be a splitting field for $X$. Suppose $X$ has a smooth point fixed by a finite abelian p-subgroup $H$ of $G$. Then $[L: K]$ is divisible by $\left[H: H_{T}\right]$ for some toral subgroup $H_{T}$ of $H$.

If $X$ is a generically free primitive $G$-variety, $K=k(X)^{G}$, and $L$ is a splitting field which is a (finite) Galois extension of $K$, then we shall refer to $\operatorname{Gal}(L / K)$ as a splitting group for $X$.

Theorem 1.2. Let $X$ be a generically free primitive $G$-variety and let $A$ be a splitting group for $X$. Suppose $X$ has a smooth point fixed by a finite abelian subgroup $H$ of $G$. Then $A$ contains an isomorphic copy of $H / H_{T}$ for some toral subgroup $H_{T}$ of $H$.

Our proofs of Theorems 1.1 and 1.2 are based on the following results of [RY]: For any finite abelian subgroup $H$ of $G$, the existence of a smooth $H$-fixed point on a (complete smooth) $G$-variety $X$ is a birational invariant of $X$. Moreover, such points survive under dominant rational $G$-equivariant maps and under certain $G$-equivariant covers; see [RY, Section 5 and Appendix]. We review and further extend these results in Section 2; see Proposition 2.2 and Theorems 2.5, 2.6 and 2.7.

Informally speaking, Theorem 1.1 (respectively, Theorem 1.2) may be viewed as a "lower bound" on a splitting field (respectively, a splitting group) of $X$. In particular, if $X$ is a vector space and $G$ acts linearly on $X$ then $X$ has a smooth $G$-fixed point (namely, the origin) and, hence, in this case Theorem 1.1 (respectively, Theorem 1.2) can be applied to every finite abelian $p$-subgroup (respectively, subgroup) $H$ of $G$. Of course, Theorems 1.1 and 1.2 are only of interest if $H$ is nontoral, since otherwise $H / H_{T}$ may be trivial.

Elementary finite abelian subgroups of algebraic groups have been extensively studied (see [BS], [Bor1], [St], [Se5]); a complete classification was obtained by Griess [Gri]. To the best of our knowledge, nonelementary 
finite abelian subgroups have not been classified. In Section 5 we apply Theorem 1.1 to a number of specific groups $G$, where we have sufficient information about the depth of certain nontoral subgroups (see Definition 4.5). In particular, for $G=E_{8}$ we give a new proof of a theorem of Serre; see Corollary 5.5. Note, however, that the examples we give in Section 5 are somewhat fragmentary, because we do not know any general results about the depth of finite abelian subgroups in exceptional groups. (Propositions 5.3 and 5.7 represent our best efforts in this direction; see also Corollary 4.10.) We hope that this question will attract the attention of group theorists in the future, and that a more complete picture will emerge.

"Upper bounds" on the degrees of splitting fields, i.e., results of the form "every $G$-variety can be split by a field extension of degree dividing $n(G)$ ", can be found in the paper [T2] of Tits. For a discussion of these results, including a table of values for $n(G)$, see Remark 4.9.

In Sections 8 and 9 we apply Theorem 1.2, with $G=\mathrm{PGL}_{n}$, to the theory of central simple algebras. Recall that an element $\alpha \in H^{1}\left(K, \mathrm{PGL}_{n}\right)$ may be (functorially) identified with an $n^{2}$-dimensional central simple $K$-algebra $D_{\alpha}$; see Example 3.1. In particular, $L / K$ is a splitting field for $\alpha$ if and only if $L$ is a splitting field for $D_{\alpha}$, i.e., $D_{\alpha} \otimes_{K} L=M_{n}(L)$. Recall that $D$ is an $H$-crossed product iff $H$ is a splitting group for $D$ and $|H|=\operatorname{deg}(D)$.

Let $\operatorname{UD}(n, k)$ be the universal division algebra of degree $n$, i.e., the division algebra generated by two generic matrices, $X=\left(x_{i j}\right)$ and $Y=\left(y_{i j}\right)$, in $\mathrm{M}_{n}\left(k\left(x_{i j}, y_{i j}\right)\right)$. Here $x_{i j}, y_{i j}$ are algebraically independent commuting variables over $k$. If the reference to $k$ is clear from the context, we shall write $\mathrm{UD}(n)$ in place of $\mathrm{UD}(n, k)$. A famous theorem of Amitsur asserts that $\operatorname{UD}(n)$ is not a crossed product if $n$ is divisible by $p^{3}$ for some prime $p$.

As an application of Theorem 1.2 we will prove the following result.

Theorem 1.3. Let $Z\left(p^{r}\right)$ be the center of the universal division algebra $\mathrm{UD}\left(p^{r}\right)$, let $K$ be a field extension of $Z\left(p^{r}\right)$ and let $D=\mathrm{UD}\left(p^{r}\right) \otimes_{Z\left(p^{r}\right)} K$. Suppose $p^{e}$ is the highest power of $p$ dividing $\left[K: Z\left(p^{r}\right)\right]$, where $e$ is a nonnegative integer and $e \leq r-1$. If $A$ is a splitting group for $D$ then

$$
p^{2 r-2 e-2}|| A \mid \text {. }
$$

In particular, if $r \geq 2 e+3$ then $D$ is a noncrossed product.

If $K=Z\left(p^{r}\right)$, i.e., $D=\mathrm{UD}\left(p^{r}\right)$, we recover a theorem of Amitsur and Tignol; see [TA1, Theorem 7.3]. If $e=0$, i.e., $D$ is a prime-to- $p$ extension of $\mathrm{UD}\left(p^{r}\right)$, we recover a theorem of Rowen and Saltman [RS, Theorem 2.1] to the effect that $D$ is not a crossed product for any $r \geq 3$.

Abelian subgroups of $\mathrm{PGL}_{n}$ carry a natural skew-symmetric form and their nontoral subgroups are isotropic with respect to this form; see Section 7. Thus symplectic modules and their Lagrangian submodules, used by Tignol and Amitsur to prove [TA1, Theorem 7.3], naturally arise in 
our setting; in particular, they will be used in the proof of Theorem 1.3 in Section 8.

It is likely that Theorem 1.3 can also be proved by an application of Amitsur's specialization technique, along the lines of [RS, Section 2] and that such a proof will go through in prime characteristic (assuming $p \nmid \operatorname{char}(k)$ ). We believe that our approach, based on the fixed points of nontoral subgroups, is of independent interest; in particular, it shows that Theorem 1.3 remains true if $\mathrm{UD}\left(p^{r}\right)$ is replaced by any central simple algebra whose corresponding $\mathrm{PGL}_{n}$-variety has points fixed by certain nontoral subgroups of $\mathrm{PGL}_{n}$; see Remark 8.4.

As another application of Theorem 1.2 with $G=\mathrm{PGL}_{n}$, we construct a noncrossed product division algebra over a "small" function field. Since the time of Amitsur's original examples, two other noncrossed product constructions have appeared in the literature, due, respectively, to JacobWadsworth $[\mathbf{J W}]$ and Brussel $[\mathbf{B r}]$. Both of these examples have the property that their centers are "smaller" and easier to describe than the center of Amitsur's "generic" example, UD $\left(p^{r}, k\right)$.

The problem we address here is one of constructing noncrossed product examples over "small" fields in the geometric setting, i.e., noncrossed products $D$ with center $K$ such that $K$ is a function field over an algebraically closed base field $k$ of characteristic 0 . Moreover, we would like "the size of $K$ ", as measured by $\operatorname{trdeg}_{k}(K)$, to be as small as possible.

Note that $\operatorname{trdeg}_{k}(K)$ cannot be $\leq 1$ by Tsen's theorem. Moreover, division algebras $D$ with $\operatorname{trdeg}_{k}(K)=2$ are conjectured to be cyclic. At the other extreme, if $D=\mathrm{UD}(n)$ is Amitsur's original noncrossed product example (with $n$ divisible by $p^{3}$ for some prime $p$ ) then $\operatorname{trdeg}_{k}(K)=n^{2}+1$.

In this paper we prove the following theorem.

Theorem 1.4. Let $p$ be a prime, $r \geq 2$ be an integer, and $k$ be an algebraically closed field of characteristic 0 . Then there exists a division algebra $D$ of degree $p^{r}$ with center $K$, such that;

(a) $K$ is a finitely generated extension of $k$ of transcendence degree 6 and;

(b) no prime-to-p extension of $D$ is a crossed product.

The idea of the proof is as follows. We show (see Section 8) that it

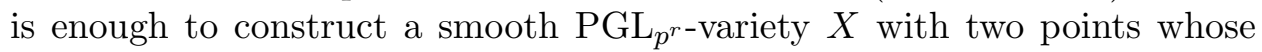
stabilizers are "incompatible" symplectic modules $\left(\mathbb{Z} / p^{r} \mathbb{Z}\right)^{2}$ and $(\mathbb{Z} / p \mathbb{Z})^{6}$; such varieties are fairly easy to construct. The difficult part is to reduce the dimension of $X / \mathrm{PGL}_{p^{r}}$ to 6 ; this is done in Section 9. Our argument there is based on a resolution result for the fixed point loci of finite abelian subgroups (we show that the fixed-point set of a finite abelian subgroup $H$ can be resolved in such way that it has a component of the minimal possible codimension, equal to rank $H$; see Theorem 9.3) and on a form of Bertini's 
theorem in the equivariant setting (Theorem 9.7). We believe Theorems 9.3 and 9.7 are of independent interest.

A.R. Wadsworth has pointed out to us that Theorem 1.4 can be proved by modifying the arguments of $[\mathbf{J W}]$. This approach, based on valuation theory, cohomology, and the Merkurjev-Suslin theorem, yields the desired result under the assumption that $p \nmid \operatorname{char}(k)$.

Throughout this paper we shall work over a fixed base field $k$ which will be assumed to be algebraically closed and of characteristic zero. The assumption that $k$ should be algebraically closed is usually not essential: Generally speaking, the problems we wish to consider (such as constructing noncrossed products or proving lower estimates on the size of splitting fields) can only become harder after passing to the algebraic closure. The characteristic zero assumption is more serious, since most of our proofs ultimately rely on canonical resolution of singularities (via Proposition 2.2 and Theorem 2.5).

Acknowledgements. We would like to thank D.J. Saltman, J.-P. Serre, and A.R. Wadsworth for helpful discussions.

\section{2. $G$-varieties.}

Preliminaries. A $G$-variety $X$ is an algebraic variety with a $G$-action. Here $G, X$ and all other algebraic objects in this paper are assumed to be defined over a fixed base field $k$. Unless otherwise specified, we shall assume that $k$ is algebraically closed and of characteristic 0 . The $G$-action on $X$ is given by a morphism $G \times X \longrightarrow X$. If the reference to the action is clear from the context, we shall write $g x$ for the image of $(g, x)$ under this map. Given $x \in X$, the stabilizer of $x$ is defined as $\{g \in G \mid g x=x\}$; we will denote this subgroup of $G$ by $\operatorname{Stab}_{G}(x)$ or $\operatorname{simply} \operatorname{Stab}(x)$ if the reference to $G$ is clear from the context.

By a morphism $X \longrightarrow Y$ of $G$-varieties, we shall mean a $G$-equivariant morphism from $X$ to $Y$. The same goes for rational morphism, isomorphism, birational morphism, etc., of $G$-varieties.

A $G$-variety $X$ is called primitive if $G$ transitively permutes the irreducible components of $X$. Equivalently, $X$ is primitive iff $k(X)^{G}$ is a field. Note that an irreducible $G$-variety is necessarily primitive and that the converse holds if $G$ is a connected group.

If $X$ is a $G$-variety then any variety $Y$ with $k(Y)=k(X)^{G}$ is called a rational quotient variety for $X$; we will often write $Y=X / G$. Note that $X / G$ is only defined up to birational isomorphism and that $X$ is a primitive $G$-variety iff $X / G$ is irreducible. The inclusion $k(Y)=k(X)^{G} \hookrightarrow$ $k(X)$ induces the rational quotient map $\pi: X \rightarrow X / G$. By a theorem of Rosenlicht $\pi^{-1}(y)$ is a single $G$-orbit for $y$ in general position in $X / G$ (see [Ro1, Theorem 2], [Ro2]). 
A $G$-variety $X$ is called generically free if $\operatorname{Stab}(x)=\{1\}$ for $x$ in general position in $X$. We will usually consider $G$-varieties that are both primitive and generically free. Up to birational isomorphism, a primitive generically free $G$-variety may be viewed as a principal $G$-bundle over $X / G$ and thus represents a class in $H^{1}(K, G)$, where $K=k(X)^{G}$; see [Po, Section 1.3]. We shall return to this connection in Section 3.

It is often convenient to have a concrete (biregular) model for $X / G$. If $G$ is a finite group then, under rather mild assumptions on $X$, we have such a model in the form of a geometric quotient, which we shall denote by $X / / G$. Here the quotient map $\pi: X \longrightarrow X / / G$ is regular and each fiber of this map is a single $G$-orbit. For a precise definition and a detailed discussion of the geometric quotient we refer the reader to [PV, Section 4.2].

Lemma 2.1. Let $G$ be a finite group and $X$ be a normal quasiprojective $G$-variety. Then:

(a) $X$ is covered by affine open $G$-invariant subsets.

(b) There exists a geometric quotient map $\pi: X \longrightarrow X / / G$.

(c) Moreover, if $X$ is projective then so is $X / / G$.

Proof. (a) By a theorem of Kambayashi, we may assume without loss of generality that $X \subset \mathbb{P}(V)$, where $V$ is a finite-dimensional vector space, and $G$-acts linearly on $X$, via a representation $G \longrightarrow \mathrm{GL}(V)$; see [Ka, Theorem 2.5] or [PV, Theorem 1.7].

We want to show that every $x \in X$ has an affine $G$-invariant neighborhood in $X$. To construct this neighborhood, choose a homogeneous polynomial $h \in k[V]$ such that $h(g x) \neq 0$ for every $g \in G$ but $h(y)=0$ for every $y \in \bar{X}-X$. After replacing $h$ by the product of $g^{*} h$ over all $g \in G$, we may assume $h$ is $G$-invariant. Now $\{z \in \bar{X} \mid h(z) \neq 0\}$ is a desired affine $G$-invariant neighborhood of $x$.

(b) Follows from part (a) and [PV, Theorem 4.14].

(c) See $[\mathbf{P V}$, Theorem 4.16].

The variety $X_{L}$. Let $X$ be a generically free primitive $G$-variety, let $K=$ $k(X)^{G}$ and let $c l(X)$ be the class of $X$ in $H^{1}(K, G)$. Suppose $L$ is a finitely generated field extension of $K$. Then $X_{L}$ is defined as the $G$-variety representing the image of $c l(X)$ under the natural map $H^{1}(K, G) \longrightarrow H^{1}(L, G)$. In other words, $\operatorname{cl}(X) \mapsto \operatorname{cl}\left(X_{L}\right)$ under this map.

To construct $X_{L}$ explicitly, let $Y \rightarrow X / G$ be a rational map such that $k(Y) / k(X / G)$ is precisely the extension $L / K$. Note that such a rational map exists because $L$ is finitely generated over $K$ and, hence, over $k$. Now we set $X_{L}=Y \times_{X / G} X$, where the $G$-action on $X_{L}$ is induced from the $G$-action on $X$; cf. [Re, Section 2.6].

We emphasize that $X_{L}$ is only defined up to birational isomorphism (of $G$ varieties). We we will often want to work with a specific model for $X_{L}$ which 
is smooth or projective or has "small" stabilizers (or all of the above). The existence of such models is guaranteed by Proposition 2.2 and Theorem 2.5.

\section{Smooth projective models for $G$-varieties.}

Proposition 2.2. Every $G$-variety is birationally isomorphic to a smooth projective $G$-variety.

Proof. Let $X$ be a $G$-variety. By [RY, Proposition 7.1], $X$ is birationally isomorphic to a complete $G$-variety. (Note that the proof of [RY, Proposition 7.1] is based on Sumihiro's equivariant completion theorem.) Thus we may assume without loss of generality that $X$ is complete.

Now by [Ka, Theorem 2.5] there exists a projective representation $G \hookrightarrow$ $\operatorname{PGL}(V)$ and a closed $G$-invariant subvariety $X^{\prime}$ of $\mathbb{P}(V)$ such that $X$ and $X^{\prime}$ are birationally isomorphic as $G$-varieties. After replacing $X$ by $X^{\prime}$, we may assume $X$ is projective. Now apply the canonical resolution of singularities theorem (see either [V, Theorem 7.6.1] or [BM, Theorem 13.2]) to $X$ to construct a smooth projective model.

Definition 2.3. We shall call an algebraic group $H$ Levi-commutative if $H$ is a semidirect product of a diagonalizable group $D$ and a unipotent group $U$, where $U \triangleleft H$ is the unipotent radical of the identity component $H_{0}$ of $H$.

We shall denote $U=R_{u}\left(H_{0}\right)$.

Lemma 2.4. Let $H$ be an algebraic group and let $H_{0}$ be the identity component of $H$. The following conditions are equivalent.

(i) $H$ is Levi-commutative,

(ii) $H / R_{u}\left(H_{0}\right)$ is commutative,

(iii) every reductive subgroup of $H$ is commutative, and

(iv) every linear representation of $H$ has 1 -dimensional $H$-invariant subspace.

Proof. The equivalence of (i), (ii) and (iii) follows from the Levi decomposition theorem (see [OV, Section 6.4]). The equivalence of (i) and (iv) is proved in [RY, Lemma A.1].

Theorem 2.5. Let $X$ be a G-variety. Then there exists a sequence of blowups

$$
X_{n} \longrightarrow X_{n-1} \longrightarrow \ldots \longrightarrow X_{0}=X
$$

such that $X_{n}$ is smooth and $\operatorname{Stab}(x)$ is Levi-commutative for every $x \in X_{n}$.

Proof. See [RY, Theorem 1.1]. 


\section{Going up and going down.}

Theorem 2.6 (Going down). Let $H$ be a Levi-commutative group (see Definition 2.3) and let $X \rightarrow Y$ be a rational map of $H$-varieties. Suppose $Y$ is complete and $X$ has a smooth point fixed by $H$. Then $Y$ has a smooth point fixed by $H$.

Proof. See [RY, Propositions 5.3 and A.2].

Theorem 2.7 (Going up). Let $H$ be a finite abelian group of prime power order $p^{n}$ and let $f: X \rightarrow Y$ be a dominant rational map of $H$-varieties. Suppose

(i) $Y$ is irreducible,

(ii) $X$ is complete,

(iii) $\operatorname{dim}(X)=\operatorname{dim}(Y)$,

(iv) $H$ has a smooth fixed point in $Y$, and

(v) $\operatorname{deg}(X / Y)$ is not divisible by $p^{e+1}$.

Then there exists a subgroup $H^{\prime}$ of $H$ such that $\left|H^{\prime}\right| \geq p^{n-e}$ and

(a) $H^{\prime}$ has a fixed point in $X$.

(b) Moreover, if $X \rightarrow Z$ is a rational map of complete $H$-varieties then $H^{\prime}$ has a fixed point in $Z$.

This theorem is a generalization of [RY, Propositions 5.5 and A.4], where $e$ is assumed to be 0. Our proof below is based on an argument of Kollár and Szabó; cf. [RY, Proposition A.4]. Our applications will only use (a); however, part (b) is needed for the inductive argument.

A convenient way to visualize the setting of Theorem 2.7 is by means of the diagram

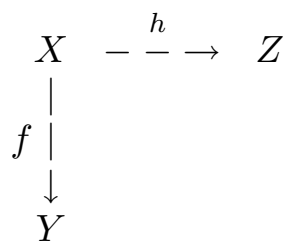

If $e=0$ the theorem allows us to lift an $H$-fixed point from $Y$ to $X$, then transport it to $Z$. (A similar but slightly weaker statement is true if $e \geq 1$.) We will make use of such diagrams in the proof.

Proof. The proof is by induction on $d=\operatorname{dim} Y$. If $\operatorname{dim} Y=0$ then $Y$ is a point, $X$ is a set of $\operatorname{deg}(X / Y)$ points, and the desired result follows from a simple counting argument.

To perform the induction step, we assume that the theorem holds whenever $\operatorname{dim}(Y) \leq d-1$. Let $y \in Y$ be a smooth fixed point, $B_{y}(Y)$ be the blowup of $Y$ at $y$, and $Y^{\prime} \subset B_{y} Y$ be the exceptional divisor. Note that $H$ acts linearly on $Y^{\prime}=\mathbb{P}^{\operatorname{dim}(Y)-1}$ and, hence, has a fixed point in $Y^{\prime}$; see 
Lemma 2.4(iv). This fixed point will be smooth because every point of $Y^{\prime}$ is smooth.

Let $X=\bigcup_{i} X_{i}$ be the decomposition of $X$ into irreducible components. It is enough to find the required fixed point in one of the components $X_{i}$ which is mapped dominantly onto $Y$; thus, we may assume that all $X_{i}$ are mapped dominantly onto $Y$.

It follows that each map $X_{i} \rightarrow B_{y} Y$ is dominant; let $\bar{X}_{i}$ be the normalization of $B_{y} Y$ in the field of rational functions on $X_{i}, \bar{X}$ be the disjoint union of all $\bar{X}_{i}$ (in other words, $\bar{X}$ is the normalization of $B_{y} Y$ in the ring $\left.k(X)=\bigoplus_{i} k\left(X_{i}\right)\right)$, and $\bar{f}: \bar{X} \longrightarrow B_{y} Y$ be the natural morphism. Clearly, $H$ acts on $\bar{X}$ and $\bar{f}$ is $H$-equivariant. Each $\bar{X}_{i}$, it is birationally isomorphic to $X_{i}$. Together these birational isomorphisms yield an $H$-equivariant birational isomorphism $\bar{X} \rightarrow X$.

Let $F_{1}, F_{2}, \ldots \subset \bar{X}$ be the divisors lying over $Y^{\prime}$. Note that even though $\bar{X}$ is not necessarily complete, each $F_{i}$ is complete since it is mapped finitely to a complete variety $Y^{\prime}$.

The group $H$ acts on the set $\left\{F_{i}\right\}$. Let $\mathcal{F}_{j}$ denote the $H$-orbits in $\left\{F_{i}\right\}$. Choose a divisor $F_{j}^{*} \in \mathcal{F}_{j}$ in each orbit. By the ramification formula (see, e.g., [L, Corollary XII.6.2]),

$$
\operatorname{deg}(X / Y)=\sum_{j}\left|\mathcal{F}_{j}\right| \cdot \operatorname{deg}\left(F_{j}^{*} / E\right) \cdot e\left(\bar{f}, F_{j}^{*}\right)
$$

where $e\left(\bar{f}, F_{j}^{*}\right)$ denotes the ramification index of $\bar{f}$ at the generic point of $F_{j}^{*}$. Since $\operatorname{deg}(X / Y)$ is not divisible by $p^{e+1},\left|\mathcal{F}_{j}\right| \cdot \operatorname{deg}\left(F_{j}^{*} / E\right)$ is not divisible by $p^{e+1}$ for some $j$. For this $j$, set $X^{\prime}=H F_{j}^{*}=\bigcup_{F_{i} \in \mathcal{F}_{j}} F_{i}$; this variety is complete since each $F_{i}$ is complete. Let $f^{\prime}: X^{\prime} \longrightarrow Y^{\prime}$ be the restriction of $\bar{f}: \bar{X} \longrightarrow B_{z}(Y)$ to $X^{\prime}$; the degree of $f^{\prime}$ is equal to $\left|\mathcal{F}_{j}\right| \cdot \operatorname{deg}\left(F_{j}^{*} / E\right)$, and hence, is not divisible by $p^{e+1}$. Let $h^{\prime}: X^{\prime} \rightarrow X$ be the restriction of $\bar{X} \rightarrow X$ to $X^{\prime}$. Note that $h^{\prime}$ is well-defined, since $\bar{X}$ is normal, $X$ is complete, and $X^{\prime}$ is a divisor in $\bar{X}$.

By our construction, $\operatorname{dim}\left(Y^{\prime}\right)=d-1$ and conditions (i) $-(\mathrm{v})$ hold for the map $f^{\prime}: X^{\prime} \rightarrow Y^{\prime}$. Applying the induction assumption to the diagram

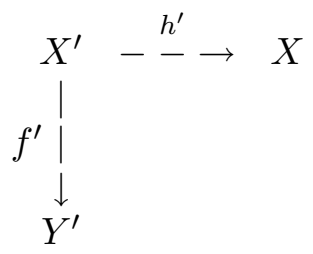


we prove part (a). Applying the induction assumption to the diagram

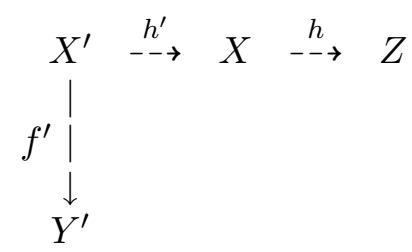

we prove part (b).

Remark 2.8. Theorems 2.6 and 2.7 are valid over an algebraically closed field of arbitrary characteristic; the proofs given above are characteristicfree.

\section{Two interpretations of $H^{1}$.}

Let $A$ be a finite-dimensional algebra over $k$. We do not assume that $A$ is commutative, associative or has an identity element. Let $K$ is a field extension of $k$. We shall say that a $K$-algebra $B$ is of type $A$ if $B_{\bar{K}} \simeq A_{\bar{K}}$, where $\bar{K}$ is the algebraic closure of $K$. (Here, as usual, $B_{\bar{K}} \stackrel{\text { def }}{=} B \otimes_{K} \bar{K}$ and $A_{\bar{K}} \stackrel{\text { def }}{=} A \otimes_{k} \bar{K}$.)

Let $G=\operatorname{Aut}_{k}(A)$. It is easy to see that $G$ is a closed subgroup of $\mathrm{GL}_{\operatorname{dim}(A)}$; thus it is an algebraic group. We now have the following bijections.

$$
H^{1}(K, G) \longleftrightarrow\left\{\begin{array}{l}
\text { Birational isomorphism classes } \\
\text { of primitive generically free } \\
G \text {-varieties } X \text { with } k(X)^{G}=K
\end{array}\right\}
$$

$\{K$-algebras of type $A\}$

The horizontal correspondence is described in [Po, Section 1.3]; the vertical one in [Se2, Section X.2] or [KMRT, Proposition 29.1]. Note that the above diagram is functorial in $K$ and that the correspondences in it are bijections of pointed sets: The identity elements of $H^{1}(K, G)$ corresponds to the "split" algebra $A_{K}$ and to the "split" variety $X=X_{0} \times G$, where $k\left(X_{0}\right)^{G}=K$; see Definition 4.1 .

In this paper we shall be primarily interested in passing back and forth between $G$-varieties and algebras of type $A$. In other words, we would like to construct an explicit correspondence $X \mapsto B$ which completes the triangle in the above diagram. Given a generically free primitive $G$-variety $X$ with $k(X)^{G}=K$, we define $B=\operatorname{RMaps}_{G}(X, A)$. Here $\operatorname{RMaps}_{G}(X, A)$ is the set of $G$-equivariant rational maps $X \rightarrow A$, where we view $A$ as a $k$-vector 
space with a $G$-action. The $k$-algebra structure on $A$ gives rise to a $K$ algebra structure on $B$ via

$$
\begin{aligned}
& \left(f_{1}+f_{2}\right)(x) \stackrel{\text { def }}{=} f_{1}(x)+f_{2}(x), \\
& \left(f_{1} f_{2}\right)(x) \stackrel{\text { def }}{=} f_{1}(x) f_{2}(x), \\
& \left(\alpha f_{2}\right)(x) \stackrel{\text { def }}{=} \alpha(x) f_{1}(x) .
\end{aligned}
$$

Here $f_{1}, f_{2} \in \operatorname{RMaps}_{G}(X, A), \alpha \in K, x$ is an element of $X$ in general position, and the operations in the right hand sides of the above formulas are performed in $A$. The correspondence $X \mapsto \operatorname{RMaps}_{G}(X, A)$ completes the triangle in the above diagram; see [Re, Proposition 8.6 and Lemma 12.3].

Example 3.1. $A=\mathrm{M}_{n}(k)$ is the algebra of $n \times n$-matrices over $k$. By a theorem of Wedderburn, $B$ is a $K$-algebra of type $\mathrm{M}_{n}$ if and only if $B$ is a central simple $K$-algebra; see e.g., [KMRT, Theorem 1.1] or [Se2, X.5, Proposition 7].

Note that $\operatorname{Aut}_{k}(A)=\mathrm{PGL}_{n}$. Thus, if $K$ is a finitely generated field extension of $k$, every central simple $K$-algebra $B$ of degree $n$ is of the form $\operatorname{RMaps}_{\mathrm{PGL}_{n}}\left(X, \mathrm{M}_{n}(k)\right)$ for some generically free irreducible $\mathrm{PGL}_{n}$-variety $X$ with $k(X)^{G}=K$. Moreover, the $G$-variety $X$ is uniquely determined up to birational isomorphism. For example, if $B=\mathrm{UD}(n)$ is the universal division algebra of degree $n$ generated by two generic matrices then $B=$ $\operatorname{RMaps}_{\mathrm{PGL}_{n}}\left(X, \mathrm{M}_{n}(k)\right)$, where $X=\mathrm{M}_{n}(k) \times \mathrm{M}_{n}(k)$ and $\mathrm{PGL}_{n}$ acts on $X$ by simultaneous conjugation. This description of $\operatorname{UD}(n)$ is due to Procesi; see [Sa, Theorem 14.16] or [Pr, Theorem 2.1].

Example 3.2. $A=\mathrm{O}$ is the 8-dimensional split Cayley algebra (otherwise known as the split octonion algebra). Then $\operatorname{Aut}(\mathbf{O})$ is the exceptional group $G_{2}$. By a theorem of Zorn, $B$ is a $K$-algebra of type $\mathbf{O}$ if and only if $B$ is a Cayley algebra over $K$. Cayley algebras are thus in natural $1-1$ correspondence with generically free irreducible $G_{2}$-varieties; see [Re, Remark 11.4], [Se3, Section 8.1] and [KMRT, Proposition 33.24].

Example 3.3. $A$ is the 27-dimensional (split) Albert algebra (otherwise known as an exceptional simple Jordan algebra) defined over $k$. Then $\operatorname{Aut}(A)$ is the exceptional group $F_{4}$. Algebras of type $A$ are precisely the Albert algebras, i.e., a 27-dimensional exceptional simple Jordan algebras; see e.g., [KMRT, p. 517], [Se3, Section 9].

Remark 3.4. The results of this section remain valid if the algebra $A$ is replaced by a more general algebraic object consisting of a vector space with a tensor on it. Such objects are called structured spaces in $[\mathbf{R e}]$. We refer the reader there for details; see also [KMRT, Section 29]. 


\section{Splitting fields.}

Definition 4.1. Let $G$ be an algebraic group and $X$ be a primitive generically free $G$-variety, $K=k(X)^{G}$ and $c l(X)=$ the class of $X$ in $H^{1}(K, G)$. We will say that $X$ is split if $X$ is birationally isomorphic to $X / G \times G$ (as a $G$-variety). Equivalently, $X$ is split if there exists a rational section $X / G \rightarrow X$ or, if $c l(X)=1$; see, e.g., [Po, 1.4.1].

A field extension $L$ of $K$ is called a splitting field of $X$ if $X_{L}$ is split. Equivalently, $L$ is a splitting field if the image of $\operatorname{cl}(X)$ under the natural map $H^{1}(K, G) \longrightarrow H^{1}(L, G)$ is trivial.

Remark 4.2. Recall that an algebraic group $G$ is called special if every generically free $G$-variety is split. Special groups were studied by Serre [Se1] and classified by Grothendieck [Gro, Theorem 3]; see also [PV, Theorem 2.8]. In particular, $\mathrm{GL}_{n}, \mathrm{SL}_{n}, \mathrm{Sp}_{2 n}$, and the additive group $\mathrm{G}_{\mathrm{a}}$ are special. Moreover, it is easy to see that if $N$ is a normal subgroup of $G$ and both $N, G / N$ are special, then so is $G$. In particular, every connected solvable group is special; cf. [PV, Section 2.6].

\section{Split $G$-varieties.}

Lemma 4.3. Let $G$ be an algebraic group, let $X$ be a split $G$-variety and let $H$ be a Levi-commutative subgroup of $G$ (see Definition 2.3). If $H$ has a smooth fixed point in $X$ then

(a) $H$ is contained in a Borel subgroup of $G$. Moreover,

(b) if $H$ is diagonalizable then it is contained in a maximal torus of $G$.

Proof. Since $X$ is split, it is birationally isomorphic to $X_{0} \times G$ for some variety $X_{0}$, where $G$ acts trivially on the first factor and by left translations on the second. Let $B$ is a Borel subgroup of $G$. Consider the rational $G$-equivariant map

$$
X \simeq X_{0} \times G \rightarrow G / B .
$$

sending $\left(x_{0}, g\right)$ to $g \bmod B$. Since $G / B$ is a complete variety, the Going Down Theorem 2.6 tells us that $H$ fixes a point of $G / B$. Consequently, $H$ is contained is a conjugate of $B$, and part (a) follows. Part (b) is immediate from part (a) and [Bor2, Theorem 10.6(5)].

Remark 4.4. Note that our proof of Lemma 4.3 relies only on the Going Down Theorem and not on Proposition 2.2. In particular, Lemma 4.3(a) is valid in arbitrary characteristic; see Remark 2.8.

Proof of Theorem 1.1. Consider the natural projection map $X_{L} \rightarrow X$ of $G$-varieties. Here $L$ is a splitting field for $X$, i.e., $X_{L}$ is split. By Proposition 2.2, we may assume without loss of generality that $X_{L}$ is smooth and projective. Let $p^{e}$ be the maximal power of $p$ dividing $[L: K]=\operatorname{deg}\left(X_{L} / X\right)$. 
By the Going Up Theorem 2.7(a), there exists a point $y \in X_{L}$ and a subgroup $H^{\prime}$ of $H$ such that (i) $\left[H: H^{\prime}\right]$ divides $p^{e}$ and (ii) $H^{\prime} \subset \operatorname{Stab}(y)$. Now (i) says that $\left[H: H^{\prime}\right]$ divides $[L: K]$ and (ii), in combination with Lemma 4.3(b), says that $H^{\prime}$ is toral.

\section{Nontoral subgroups.}

Definition 4.5. Let $G$ be an algebraic group and let $H$ be an abelian $p$ subgroup of $G$. The depth of $H$ is defined as the smallest integer $i$ such that $H$ has a toral subgroup of index $p^{i}$.

Recall that a prime number $p$ is called a torsion prime for $G$ if $G$ has a nontoral abelian $p$-subgroup $H$, i.e., an abelian $p$-subgroup $H$ of depth $\geq 1$. Following $[\mathbf{S e} 5,1.3]$ we will denote the set of torsion primes for $G$ by $\operatorname{Tors}(G)$.

Remark 4.6. Torsion primes have been extensively studied; see [Bor1], [SS], [St] and [Se5]. In particular, Tors $(G)=$ Tors $\left(G^{\prime}\right)$ if $G^{\prime}$ is a derived subgroup of $G$; see [Se5, 1.3.2]. If $f: \bar{G} \longrightarrow G$ is the universal cover of $G$ then $\operatorname{Tors}(G)$ is the union of Tors $(\bar{G})$ and the set of prime divisors of $\operatorname{Ker}(f)$; see [Se5, 1.3.3].

For simply connected simple groups the torsion primes are given by the following table:

$$
\begin{array}{lc}
\frac{\text { Type of } G}{A_{n} \text { or } C_{n}} & \frac{\text { Tors }(G)}{\emptyset} \\
B_{n}(n \geq 3), D_{n}(n \geq 4) \text { or } G_{2} & \{2\} \\
F_{4}, E_{6}, \text { or } E_{7} & \{2,3\} \\
E_{8} & \{2,3,5\}
\end{array}
$$

For details see [Bor1, Proposition 4.4], [St, Corollary 1.13] or [Se5, 1.3.3].

Using the terminology of Definition 4.5, we can rephrase Theorem 1.1 as follows.

Theorem 4.7. Let $G$ be an algebraic group, $H$ be a finite abelian p-subgroup of $G$ of depth $d, X$ be a generically free $G$-variety and $K=k(X)^{G}$. If $X$ has a smooth $H$-fixed point and $L / K$ is a splitting field of $X$ then $p^{d} \mid[L: K]$.

In particular, if $X=V$ is a generically free linear representation of $G$ then $[L: K]$ is divisible by every torsion prime of $G$.

Let $G$ be an algebraic group and let $S$ be a special subgroup containing $G$. (For example, $S$ can be taken to be $\mathrm{GL}_{n}, \mathrm{SL}_{n}$ or $\mathrm{Sp}_{2 n}$.) We shall view $S$ as a $G$-variety with respect to the left multiplication action; it is easy to see that this variety is generically free. 
Corollary 4.8. Let $G$ be an algebraic group, $H$ be a finite abelian p-subgroup of $G$ of depth $d$ and $S$ be a special group containing $G$, as above. Suppose $K=k(S)^{G}$ and $L / K$ is a splitting field for $S$ (as a G-variety). Then $p^{d} \mid[L: K]$.

Proof. Let $\bar{S}$ be a smooth projective model of $S$ (as an $S$-variety); see Proposition 2.2. Let $V$ be a generically free linear representation of $S$. Since $S$ is special, $V$ is split as an $S$-variety. Thus there exists an $S$-equivariant dominant rational map $f: V \rightarrow S \simeq \bar{S}$. Since $G \subset S$, we can view $f: V \rightarrow \bar{S}$ as a dominant rational map of $G$-varieties. Since $V$ has a $H$-fixed point, the Going Down Theorem 2.6 tells us that so does $\bar{S}$. Applying Theorem 4.7 to the smooth variety $X=\bar{S}$, we conclude that $p^{d} \mid[L: K]$, as claimed.

Remark 4.9. Let $G$ be a simple group. A theorem of Tits asserts that every $G$-variety $X$ can be split by an extension $L / K$, where $K=k(X)^{G}$ and every prime factor of $[L: K]$ lies in $\operatorname{Tor} s(G)$; see [Se3, 2.3]. This gives a partial converse to Theorem 4.7 .

More precisely, the results of [T2] show that every $G$-variety can be split by an extension of degree dividing $n(G)$, where $n(G)$ is given by the following table.

$\begin{array}{ccc}\text { Type } & \text { Simply Connected } & \text { Not Simply Connected } \\ A_{n} & 1 & n+1 \\ B_{n} & 2^{\sup (1, n-4)} & 2^{n} \\ C_{n} & 1 & 2^{v_{2}(n)+1} \\ D_{n} & 2^{\sup (1, n-5)} & 2^{v_{2}(n)+n} \\ G_{2} & 2 & - \\ F_{4} & 6 & - \\ E_{6} & 6 & 2 \cdot 3^{4} \\ E_{7} & 12 & 2^{5} \cdot 3 \\ E_{8} & 2^{7} \cdot 3^{3} \cdot 5 & -\end{array}$

Here $G$ is an almost simple group of the indicated type (recall that $G$ is almost simple if the center $Z(G)$ is finite and $G / Z(G)$ is simple) and $v_{2}(m)$ denotes the highest power of 2 dividing $m$. 
Note that the terminology of [T2] is somewhat different from ours. A primitive $G$-variety $X$ corresponds to a group of inner type over $k(X)^{G}$ (and if $G$ is simply connected, then of strongly inner type). With these conventions, the entries for all group types other than $E_{8}$ come directly from [T2, Proposition A1] or from [T2, Propositions 1 and 2].

Our entry for $E_{8}$ follows from [T2, Corollaire 2] and the fact that $n(G)$ may be taken to be the degree of a splitting field for one particular "generic" $E_{8}$-variety; see [T1, Proposition 8]. (Recall that we are working in characteristic zero.) In fact [T2, Corollaire 2] implies that $n\left(E_{8}\right)$ may be taken to be one of the numbers $2^{7} \cdot 3 \cdot 5,2^{6} \cdot 3^{2} \cdot 5$ or $2^{4} \cdot 3^{3} \cdot 5$ (it is not currently known which one). The entry for $n\left(E_{8}\right)$ in our table is the least common multiple of these three numbers.

Combining the above-mentioned results of [T2] with Theorem 4.7, we obtain the following upper bound on the depth of abelian $p$-subgroups of quasi-simple algebraic groups.

Corollary 4.10. Let $G$ be an almost simple algebraic group and let $H$ be a finite abelian p-subgroup of $G$ of depth $d$ (not necessarily elementary). Then $p^{d}$ divides the number $n(G)$ given in Remark 4.9 .

\section{Examples.}

In this section we illustrate Theorem 1.1 for several classes of groups. The application of Theorem 1.1 to the case $G=\mathrm{PGL}_{n}$ will come up later, after we discuss the nontoral subgroups of $\mathrm{PGL}_{n}$ in Section 7; see Lemma 8.1 below.

Orthogonal groups: splitting fields of quadratic forms. Let $K$ be a finitely generated field extension of $k$. Recall that quadratic forms $q$ over $K$ are in $1-1$ correspondence with primitive generically free $O_{n}$-varieties $X$ such that $k(X)^{O_{n}}=K$; see [Se4, III. Appendix 2.2] or [KMRT, Section $29 \mathrm{E}]$. In particular, a field extension $L / K$ splits the form if and only if it splits the corresponding variety.

Proposition 5.1. Let $q=\left\langle a_{1}, \ldots, a_{n}\right\rangle$ be a quadratic form over $K$. Then

(a) there exists a splitting field $L$ for $q$ such that $L / K$ is a Galois extension with $\operatorname{Gal}(L / K)=(\mathbb{Z} / 2 \mathbb{Z})^{l}$ for some $l \leq\left[\frac{n+1}{2}\right]$.

(b) Suppose $a_{1}, \ldots, a_{n}$ are algebraically independent variables over $k, K=$ $k\left(a_{1}, \ldots, a_{n}\right)$ and $L / K$ is a splitting field for $q$. Then $[L: K]$ is divisible by $2^{\left[\frac{n+1}{2}\right]}$.

Proof. (a) Suppose $n=2 m$ is even. Let

$$
L=K\left(\sqrt{-a_{2} / a_{1}}, \ldots, \sqrt{-a_{2 m} / a_{2 m-1}}\right) .
$$


Note that $L$ is a Galois extension of $K$ and $\operatorname{Gal}(L / K)=(\mathbb{Z} / 2 \mathbb{Z})^{l}$ for some $l \leq m$. Moreover, since $\left\langle a_{2 i-1}, a_{2 i}\right\rangle \simeq\left\langle a_{2 i-1},-a_{2 i-1}\right\rangle$ for $i=1, \ldots, m$,

$$
q \simeq\left\langle a_{1},-a_{1}\right\rangle \oplus \cdots \oplus\left\langle a_{2 m-1},-a_{2 m-1}\right\rangle \simeq 0
$$

in the Witt ring of $L$. This shows that $q$ splits over $L$.

If $n=2 m+1$ is odd then a similar argument shows that

$$
L=k\left(\sqrt{-a_{2} / a_{1}}, \ldots, \sqrt{-a_{2 m} / a_{2 m-1}}, \sqrt{a_{2 m+1}}\right)
$$

is a splitting field for $q$ with $\operatorname{Gal}(L / K)=(\mathbb{Z} / 2 \mathbb{Z})^{l}$ and $l \leq m+1$.

(b) Consider the $O_{n}$-variety $X=\mathrm{M}_{n}$, with $O_{n}$ acting (linearly) on $X$ by left multiplication. Recall that $k(X)^{O_{n}}=k\left(b_{i j} \mid 1 \leq i \leq j \leq n\right)$, where $b_{i j}(x)$ is the dot product of the $i$ th and the $j$ th columns of the matrix $x \in \mathrm{M}_{n}$; see, e.g., [DC, Section 2.10] or [Re, Lemma 6.4]. Note that since $\operatorname{dim}\left(X / O_{n}\right)=\operatorname{dim}(X)-\operatorname{dim}\left(O_{n}\right)=\frac{n(n+1)}{2}$, the generators $b_{i j}$ are algebraically independent over $k$. The quadratic form corresponding to $X$ is the "generic form" $q_{X}=\sum_{i \leq j} b_{i j} x_{i} x_{j}$ defined over $k\left(b_{i j}\right)$. Let $Y$ be the subvariety of $X$ consisting of $n \times n$-matrices with mutually orthogonal columns. Then $Y$ is irreducible (see [Re, Example 3.10]) and the quadratic form corresponding to $Y$ is the "generic diagonal" form $q=\sum_{i=1}^{n} a_{i} x_{i}^{2}$ which appears in the statement of part (b). Here $a_{i}=b_{i i}$ and $q$ is defined over $K=k\left(a_{1}, \ldots, a_{n}\right)$. We can now view the usual orthogonalization process in $k^{n}$ as an $O_{n}$-equivariant rational map $f: X \rightarrow Y$. That is, we view a matrix $x \in \mathrm{M}_{n}$ as a collection of $n$ column vectors. To construct $f(x) \in Y$, we apply the orthogonalization process to this collection; the resulting $n$ mutually orthogonal vectors form the columns of $f(x)$ (see [Re, Example 3.10] for details).

Note that the point $0_{n \times n}$ is a smooth point of $X$ fixed by all of $O_{n}$; here $0_{n \times n}$ is the $n \times n$ zero matrix. Let $Y^{\prime}$ be a smooth projective model of $Y$ (see Proposition 2.2); we can thus think of $f$ as an $O_{n}$-equivariant rational map $X \rightarrow Y^{\prime}$. Let $H \simeq(\mathbb{Z} / 2 \mathbb{Z})^{n}$ be the diagonal subgroup of $O_{n}$. By the Going Down Theorem 2.6, $Y^{\prime}$ has an $H$-fixed point. This point is smooth because every point of $Y^{\prime}$ is smooth. Since $L$ splits $q$, it splits the $O_{n}$-variety $Y$ or, equivalently, the $O_{n}$-variety $Y^{\prime}$. Thus Theorem 4.7 tells us that $2^{d} \mid[L: K]$, where $d$ is the depth of $H$. Since the dimension of any maximal torus in $O_{n}$ is $\left[\frac{n}{2}\right], d \geq n-\left[\frac{n}{2}\right]=\left[\frac{n+1}{2}\right]$, as claimed.

The same argument with the group $S O_{n}$ in place of $O_{n}$ yields the following variant of Proposition 5.1. Note that elements $H^{1}\left(K, S O_{n}\right)$ represent equivalence classes of quadratic forms of determinant 1; cf. [Re, Example 8.4(b)] or [KMRT, (29.29)].

Proposition 5.2. Let $K=k\left(a_{1}, \ldots, a_{n}\right), q=\left\langle a_{1}, \ldots, a_{n}\right\rangle$ be a quadratic form of determinant 1 over $K$. Then: 
(a) There exists a splitting field $L$ for $q$ such that $L / K$ is a Galois extension with $\operatorname{Gal}(L / K)=(\mathbb{Z} / 2 \mathbb{Z})^{l}$ for some $l \leq\left[\frac{n-1}{2}\right]$.

(b) Suppose $a_{1}, \ldots, a_{n-1}$ are algebraically independent variables over $k$, $a_{n}=\left(a_{1} \ldots a_{n-1}\right)^{-1}$, and $L / K$ is a splitting field for $q$. Then $[L$ : $K] \geq 2^{\left[\frac{n-1}{2}\right]}$.

Exceptional groups $G_{2}, F_{4}, 3 E_{6}$ and $2 E_{7}$. Let $V$ be a generically free linear representation of $G$, let $K=k(V)^{G}$ and let $L / K$ be a splitting field of $V$. Theorem 4.7 tells us that $[L: K]$ is divisible by 2 if $G=G_{2}$ and by 6 if $G=F_{4}, 3 E_{6}$ or $2 E_{7}$. (Here $3 E_{6}$ and $2 E_{7}$ denote the simply connected groups of type $E_{6}$ and $E_{7}$ respectively.) If $G=G_{2}, F_{4}$ or $3 E_{6}$ then this result is sharp. In other words, $V$ can be split by $L / K$ such that $[L: K]$ equals 2 , if $G=G_{2}$ and 6 , if $G=F_{4}$ or $3 E_{6}$; see Remark 4.9 .

Exceptional group $E_{8}$. Recall that by a theorem of Adams [Ad] $E_{8}$ has two maximal elementary abelian 2-subgroups (up to conjugacy): $D(T)$ of rank 9 and $E C^{8}$ of rank 8 . Here we are following the notational conventions of [Ad, Section 2]; in particular, $D(T)$ means "double 2-torus" and $E C^{8}$ means "exotic candidate of rank 8". By construction $D(T)$ has depth 1.

Proposition 5.3. The subgroup $E C^{8} \simeq(\mathbb{Z} / 2 \mathbb{Z})^{8}$ of $E_{8}$ has depth 2 .

Our proof of this proposition uses the theory of quadratic forms over $\mathbb{Z} / 2 \mathbb{Z}$. Recall that if $q$ is a quadratic form on $V=(\mathbb{Z} / 2 \mathbb{Z})^{m}$, the associated symmetric (or, equivalently, skew-symmetric) bilinear form $b_{q}: V \times V \longrightarrow V$ is defined by $b_{q}(v, w)=q(v+w)-q(v)-q(w)$. Note that the "usual" relationship between $q$ and $b_{q}$ breaks down in characteristic 2: In particular, $b_{q}(v, v)=0$ for any $v \in V$. The kernel of $b_{q}$ is called the radical of $q$.

We shall say that $v \in V$ is an anisotropic vector for $q$ if $q(v)=1$ and an isotropic vector if $q(v)=0$. In the sequel we shall be interested in counting the number of anisotropic vectors for a given form $q$. This is not a difficult task (at least in principle) because $q$ can always be written as a direct sum of quadratic forms of dimension 1 and 2 (see, e.g., [Pf, Theorem 1.4.3]), and if $q=r \oplus s$ then a simple counting argument shows that

$$
\begin{aligned}
& \left|q^{-1}(0)\right|=\left|r^{-1}(0)\right| \cdot\left|s^{-1}(0)\right|+\left|r^{-1}(1)\right| \cdot\left|s^{-1}(1)\right| \\
& \left|q^{-1}(1)\right|=\left|r^{-1}(1)\right| \cdot\left|s^{-1}(0)\right|+\left|r^{-1}(0)\right| \cdot\left|s^{-1}(1)\right| .
\end{aligned}
$$

Lemma 5.4. Let $q$ be a quadratic form on $V=(\mathbb{Z} / 2 \mathbb{Z})^{7}$. Suppose the radical of $q$ has dimension 1 . Then $q$ has 56,64 or 72 anisotropic vectors in $V$.

Proof. Write $q \simeq q_{1} \oplus q_{2} \oplus q_{3} \oplus\langle e\rangle$, where $q_{1}, q_{2}$ and $q_{3}$ are regular 2dimensional quadratic forms and $e=0$ or 1; see, e.g., [Pf, Theorem 1.4.3]. (Here, the 1 -dimensional form $\langle e\rangle$ is the radical of $q$.) Note that over $\mathbb{Z} / 2 \mathbb{Z}$ 
there are only two classes of regular 2-dimensional quadratic forms: The hyperbolic form $h$ given by $h\left(x_{1}, x_{2}\right)=x_{1} x_{2}$ and the anisotropic form $a\left(x_{1}, x_{2}\right)=x_{1}^{2}+x_{2}^{2}+x_{1} x_{2}$. Since $a \oplus a \simeq h \oplus h$ (see [Pf, Example 2.4.5]), we may assume without loss of generality that $q_{2}=q_{3}=h$.

Case 1. $e=1$. Using (5.1) it is easy to see that if $q_{0}$ is any quadratic form on $(\mathbb{Z} / 2 \mathbb{Z})^{m}$ then $q=q_{0} \oplus\langle 1\rangle$ has exactly $2^{m}$ anisotropic vectors in $(\mathbb{Z} / 2 \mathbb{Z})^{m+1}$. In our situation $q_{0}=q_{1} \oplus q_{2} \oplus q_{3}$ and $m=6$; thus we conclude that $\left|q^{-1}(1)\right|=64$. From now on we shall assume that $e=0$.

Case 2. $q=h \oplus h \oplus h \oplus\langle 0\rangle$. We apply (5.1) to this form recursively. Since $\left|h^{-1}(0)\right|=3$ and $\left|h^{-1}(1)\right|=1$, we obtain $\left|q^{-1}(1)\right|=56$.

Case 3. $q=a \oplus h \oplus h \oplus\langle 0\rangle$. We note that $\left|a^{-1}(0)\right|=1$ and $\left|a^{-1}(1)\right|=3$, and apply (5.1) recursively, to conclude that $\left|q^{-1}(1)\right|=72$.

This completes the proof of the lemma.

Proof of Proposition 5.3. Recall that $E C^{8}=A_{1} \times A_{2} \subset G_{2} \times F_{4} \subset E_{8}$, where $A_{1}=(\mathbb{Z} / 2 \mathbb{Z})^{3}$ is the unique (up to conjugacy) nontoral abelian 2-subgroup of $G_{2}$ and $A_{2}$ is the unique (again, up to conjugacy) nontoral abelian 2subgroup of $F_{4}$; see [Gri, Theorem 2.17]. Thus, $A_{1}$ has a subgroup of index 2 which is toral in $G_{2}$, and $A_{2}$ has a subgroup of index 2 which is toral in $F_{4}$. Taking a direct product of these toral subgroups, we construct a subgroup of $E C^{8}$ of index 4 which is toral in $G_{2} \times F_{4}$ and, hence, in $E_{8}$. This proves that the depth of $E C^{8}$ is $\leq 2$.

It remains to show that the depth of $E C^{8}$ is $\geq 2$. Recall that elements of $E_{8}$ of order 2 fall into two conjugacy classes: class $A$ and class $B$; cf. [Ad, Section 5] or [Gri, (2.14)]. If $T$ is a maximal torus in $E_{8}$ and $T_{(2)}=\{t \in$ $\left.T: t^{2}=1\right\}$ then we have a naturally defined $W_{E_{8}}$-invariant quadratic form $q$ on $T_{(2)} \simeq(\mathbb{Z} / 2 \mathbb{Z})^{8}$; see [Gri, Definition 2.15]. By [Gri, Lemma 2.16] this form is nonsingular and has maximal Witt index; moreover, an element $x$ of $T_{(2)}$ is of type $A$ in $E_{8}$ if $q(x)=1$ and of type $B$ if $q(x)=0$.

In particular, of the 255 nonidentity elements of $T_{(2)}, 120$ are of type $A$ and 135 are of type $B$. On the other hand, of the 255 nonidentity elements of $E C^{8}, 56$ are of type $A$ and 199 are of type $B$; see [Ad, Section 5].

We now proceed to prove that the depth of $E C^{8}$ is $\geq 2$. Assume, to the contrary, that $E C^{8}$ has a toral subgroup $U$ of rank 7 . Since $q$ is nonsingular (i.e., the associated symplectic form $b_{q}$ is nondegenerate) on $T_{(2)}$, the radical of $q_{\mid U}$ is of dimension $\leq 1$. On the other hand, since $\operatorname{dim}(U)$ is odd, the radical of $q_{\mid U}$ cannot be trivial; thus it has dimension exactly 1 . By Lemma 5.4, $q$ has at least 56 anisotropic vectors in $U$, i.e., $U$ has at least 56 elements of type $A$. On the other hand, $E C^{8}$ has exactly 56 elements of type $A$. We therefore conclude that every element of type $A$ lies in $U$. We claim that this is impossible because the elements of type $A$ generate $E C^{8}$. This contradiction will complete the proof of the proposition. 
To prove the claim, recall that $E C^{8}=A_{1} \times A_{2}$, where $A_{1} \simeq(\mathbb{Z} / 2 \mathbb{Z})^{3}$ lies in $G_{2}$ and $A_{2}=(\mathbb{Z} / 2 \mathbb{Z})^{5}$ lies in $F_{4}$, as above. Moreover, $A_{2}$ has a subgroup $R$ of order 4 (called the radical of $E C^{8}$ ) such that

$$
S=\left(A_{2}-R\right) \cup\left(A_{1} R-R\right)
$$

is precisely the set of elements of $E C^{8}$ of type $A$; see [Gri, Theorem 2.17]. We want to show that $\langle S\rangle=E C^{8}$. Indeed, $A_{2}-R$ contains 28 of the 32 elements of $A_{2}$; these elements clearly generate all of $A_{2}$. Thus $A_{2} \subset\langle S\rangle$. In particular, $R \subset\langle S\rangle$. Now $R$, together with $A_{1} R$ generate $A_{1}$. We thus conclude that both $A_{1}$ and $A_{2}$ lie in $\langle S\rangle$. This proves that $E C^{8}=A_{1} \times A_{2}=$ $\langle S\rangle$, as claimed.

We are now ready to give an alternative proof of a theorem of Serre.

Corollary 5.5 (Serre, see [T1, Proposition 9, p. 30] or [T2, p. 1132]). Suppose $E_{8} \hookrightarrow S$, where $S=\mathrm{GL}_{n}, \mathrm{SL}_{n}$ or $\mathrm{Sp}_{2 n}$ for some $n$. We shall view $S$ as an $E_{8}$-variety via the left multiplication action. Suppose $K=k(S)^{E_{8}}$ and $L / K$ is a splitting field of $S$. Then $[L: K]$ is divisible by 60 .

Proof. Recall 2 and 3 are torsion primes of $E_{8}$, i.e., $E_{8}$ has an abelian 3subgroup and an abelian 5-subgroup, both of depth $\geq 1$. Moreover, by Proposition 5.3, $E_{8}$ contains an abelian 2-subgroup of depth 2. Thus Corollary 4.8 tells us that $[L: K]$ is divisible by $2^{2} \cdot 3 \cdot 5=60$.

Exceptional group $E_{7}$ (adjoint). We will now show that the (adjoint) group $E_{7}$ has an elementary abelian 2 -subgroup of depth $\geq 2$. We begin with the following lemma.

Lemma 5.6. Let $f: G \longrightarrow G^{\prime}$ be a surjective homomorphism of algebraic groups, such that $\operatorname{Ker}(f)$ is special. Suppose $H$ is a finite abelian subgroup of $G$ and $f(H)$ is toral in $G^{\prime}$. Then $H$ is toral in $G$.

Proof. Suppose $f(H) \subset T^{\prime} \subset G^{\prime}$, where $T^{\prime}$ be a torus in $G^{\prime}$. Denote $f^{-1}\left(T^{\prime}\right)$ by $S$. Then $H \subset S \subset G$. Moreover, since both $\operatorname{Ker}(f)$ and $S / \operatorname{Ker}(f) \simeq T^{\prime}$ are special, we conclude that $S$ is special as well; see Remark 4.2. This means that $H$ is toral in $S$ (see e.g., [Se5, 1.5.1] or Example 6.6); hence, $H$ is toral in $G$.

Proposition 5.7. The (adjoint) group $E_{7}$ has an elementary abelian 2subgroup of depth $\geq 2$.

Our proof uses the idea of Adams (see [Ad, Introduction]) to study nontoral 2-subgroups in groups of type $E_{7}$ by embedding $2 E_{7}$ into $E_{8}$.

Proof. Let $E C^{8}$ be a maximal elementary abelian subgroup of $E_{8}$ of rank 8, as in Proposition 5.3. As we mentioned in the proof of that proposition, $E C^{8}$ has 56 elements of type $A$ (in $E_{8}$ ). Let $x$ be one of these 56 elements. Denote 
the centralizer $C_{E_{8}}(x)$ by $C$. Note that $E C^{8} \subset C$. Moreover, $C \simeq 2 A_{1} E_{7}$; see [Gri, p. 280]. Thus there is an exact sequence

$$
\{1\} \longrightarrow \mathrm{SL}_{2} \longrightarrow C \stackrel{f}{\longrightarrow} E_{7} \longrightarrow\{1\} .
$$

We claim that $f\left(E C^{8}\right)$ has depth $\geq 2$ in $E_{7}$. Indeed, assume the contrary. Then $f\left(E C^{8}\right)$ contains a subgroup $H^{\prime}$ of index 2 which is toral in $E_{7}$. By Lemma 5.6, $H=f^{-1}\left(H^{\prime}\right) \cap E C^{8}$ is toral in $C$ and thus in $E_{8}$. Since $H$ is a toral subgroup of index 2 in $E C^{8}$, this implies that $E C^{8}$ has depth $\leq 1$, contradicting Proposition 5.3.

We can now prove an analogue of Corollary 5.5 for $E_{7}$.

Corollary 5.8. Suppose $E_{7} \hookrightarrow S$, where $S=\mathrm{GL}_{n}, \mathrm{SL}_{n}$ or $\mathrm{Sp}_{2 n}$ for some $n$. We shall view $S$ as an $E_{7}$-variety via the left multiplication action. Suppose $K=k(S)^{E_{7}}$ and $L / K$ is a splitting field of $S$. Then $[L: K]$ is divisible by 12 .

Proof. Recall $E_{7}$ has a nontoral abelian 3-subgroup, i.e., a 3-subgroup of depth $\geq 1$; see, e.g., [Gri]. Moreover, by Proposition 5.7, $E_{7}$ contains an abelian 2 -subgroup of depth $\geq 2$. Thus Corollary 4.8 tells us that $[L: K]$ is divisible by $2^{2} \cdot 3=12$.

\section{Splitting groups.}

\section{Definition and first examples.}

Definition 6.1. Let $X$ be a generically free primitive $G$-variety and let $K=k(X)^{G}$. We shall say that a finite group $A$ is a splitting group for $X$ if there exists a splitting field $L$ for $X$ such that $L / K$ is (finite) Galois and $\operatorname{Gal}(L / K)=A$.

Example 6.2. Let $G$ be a finite group and let $X$ be a generically free irreducible $G$-variety. Then $G$ is a splitting group for $X$.

Proof. Suppose $L=k(X)$ and $K=k(X)^{G}$. Then $k\left(X_{L}\right)=L \otimes_{K} L=$ $L \oplus \cdots \oplus L(|G|=[L: K]$ times $)$. In other words, up to birational equivalence, $X_{L}$ is the disjoint union of $|G|$ copies of $X$ and $G$ acts on $X_{L}$ by permuting these copies. Consequently, $X_{L}$ is split as a $G$-variety and $G=\operatorname{Gal}(L / K)$ is a splitting group.

Example 6.3. Let $G$ be a (connected) semisimple group, and let $W$ be the Weyl group of $G$. Then every irreducible generically free $G$-variety $X$ has a splitting group which is isomorphic to a subgroup of $W$.

Proof. Let $X$ be a generically free irreducible $G$-variety and let $\pi: X \rightarrow-\rightarrow$ $X / G$ be the rational quotient map. An irreducible subvariety $S$ of $X$ is called a Galois section if $G S$ is dense in $X$, i.e., $\pi_{\mid S}$ is dominant, and the 
field extension $k(S) / k(X)^{G}$ induced by $\pi$, is a finite Galois extension. We shall denote the group $\operatorname{Gal}\left(k(S) / k(X)^{G}\right)$ by $\operatorname{Gal}(S)$.

A theorem of Galitskii asserts that every $G$-variety $X$ has a Galois section $S$; see [Ga]. Moreover, by [Po, Remark 1.6.3] $S$ can be chosen so that $\operatorname{Gal}(S)$ is isomorphic to a subgroup $H$ of $W$. It is easy to see that in order to split $X$ as a $G$-variety it is sufficient to split $S$ as a $\operatorname{Gal}(S)$-variety. Now Example 6.2 tells us that $H$ is a splitting group for $X$.

If $G$ is a connected but not necessarily semisimple then the assertion of Example 6.3 remains true if we define $W$ as the Weyl group of $G_{s s}=$ $G / R(G)$, where $R(G)$ is the radical of $G$.

Two elementary lemmas from group theory. Our next goal is to prove Theorem 1.2. We begin with two elementary lemmas.

Lemma 6.4. Let $P$ be a finite abelian group.

(a) Every quotient group of $P$ is isomorphic to a subgroup of $P$.

(b) Every subgroup of $P$ is isomorphic to a quotient group of $P$.

Proof. Suppose $Q$ is a quotient group of $P$. Then every character of $Q$ lifts to a character of $P$. This gives an inclusion $Q^{*} \hookrightarrow P^{*}$ of dual groups. Since $Q^{*} \simeq Q$ and $P^{*} \simeq P$, part (a) follows. Part (b) is proved in a similar manner.

Lemma 6.5. Suppose $A$ and $B$ are (abstract) groups and $A \times B$ acts on a set $Z$. Let $W$ be the set of $A$-orbits in $Z$ and let $f: Z \longrightarrow W$ be the natural projection. Assume $z \in Z$ and $w=f(z)$. Then:

(a) $\operatorname{Stab}_{B}(z)$ is a normal subgroup of $\operatorname{Stab}_{B}(w)$.

(b) Let $S=\operatorname{Stab}_{A \times B}(z)$. Then $\operatorname{Stab}_{B}(z)$ is normal in $S$ and $S / \operatorname{Stab}_{B}(z)$ is isomorphic to a subgroup of $A$; we shall denote this subgroup by $A_{0}$.

(c) $\operatorname{Stab}_{B}(w) / \operatorname{Stab}_{B}(z)$ is isomorphic to a quotient of $A_{0}$.

Proof. (a) Suppose $b \in \operatorname{Stab}_{B}(w)$. Since the actions of $A$ and $B$ on $Z$ commute, $f(b z)=b f(z)=b w=w$. Consequently, $b z=a z$ for some $a \in A$ and thus

$$
b \operatorname{Stab}_{B}(z) b^{-1}=\operatorname{Stab}_{B}(b z)=\operatorname{Stab}_{B}(a z)=\operatorname{Stab}_{B}(z) .
$$

This proves part (a).

Let $\pi_{A}$ and $\pi_{B}$ be, respectively, the natural projections $A \times B \longrightarrow A$ and $A \times B \longrightarrow B$.

(b) The kernel of the map $\pi_{A}: S \longrightarrow A$ is $S \cap B=\operatorname{Stab}_{B}(z)$, and part (b) follows.

(c) Note that $b \in \operatorname{Stab}_{B}(w)$ if and only if $b z=a z$ for some $a \in A$ or, equivalently, if $\left(a^{-1}, b\right) \in S$ for some $a \in A$. In other words, $\operatorname{Stab}_{B}(w)=$ $\pi_{B}(S)$. Consequently, we have a surjective homomorphism

$$
\pi_{B}: A_{0}=S / \operatorname{Stab}_{B}(z) \longrightarrow \operatorname{Stab}_{B}(w) / \operatorname{Stab}_{B}(z) .
$$


This completes the proof of part (c).

Proof of Theorem 1.2. Let $K=k(X)^{G}$ and let $L / K$ be a Galois extension such that $\operatorname{Gal}(L / K)=A$ and $X_{L}$ is split. Note that $A \times G$ acts rationally on $X_{L}$. By a theorem of Rosenlicht (see [Ro1, Theorem 1]), we can choose a birational model for $X_{L}$ so that this action becomes regular. Moreover, after applying Proposition 2.2 and Theorem 2.5 to $X_{L}$, we may assume that

(i) $X_{L}$ is smooth and projective, and

(ii) for every $z \in X_{L}, \operatorname{Stab}_{A \times G}(z)$ is Levi-commutative (see Definition 2.3).

Note that by our construction the map $h: X_{L} \rightarrow X$ is a rational quotient map for the $A$-action on $X_{L}$. Since $A$ is a finite group and $X_{L}$ is projective, there exists a geometric quotient map $f: X_{L} \longrightarrow W=X_{L} / / A$ for the $A$-action on $X_{L}$ with $W$ projective; see Lemma 2.1. Note that by the universal property of categorical (and, hence, geometric) quotients, the $G$-action on $X_{L}$ descends to $W$; by our construction, $W$ and $X$ are birationally isomorphic as $G$-varieties. Applying the Going Down Theorem 2.6 to the birational isomorphism $X \stackrel{\simeq}{\sim} \rightarrow W$, we conclude that $W$ has a $H$-fixed point. Denote this point by $w$.

Now choose $z \in f^{-1}(w) \in X_{L}$ and apply Lemma 6.5 with $Z=X_{L}$ where we view $Z$ as an $A \times H$-variety via the obvious inclusion of $A \times$ $H$ in $A \times G$. By Lemma 6.5(b), $A$ has a subgroup $A_{0} \simeq S / \operatorname{Stab}_{H}(w)$, where $S=\operatorname{Stab}_{A \times H}(z)$. Note that $S$ is a finite subgroup of $\operatorname{Stab}_{A \times G}(z)$, and $\operatorname{Stab}_{A \times G}(z)$ is Levi-commutative by our construction (see condition (ii) above). We conclude that $S$ is abelian; see Lemma 2.4. Thus $A_{0}$ is also abelian.

By Lemma 6.5(c), $A_{0}$ has a quotient of the form $\operatorname{Stab}_{H}(w) / \operatorname{Stab}_{H}(z)=$ $H / \operatorname{Stab}_{H}(z)$. Denote $\operatorname{Stab}_{H}(z)$ by $H^{\prime}$. Since $Z=X_{L}$ is split, Lemma 4.3(b) says that $H^{\prime}$ is toral in $G$. Thus $H / H^{\prime}$ is a quotient of $A_{0}$, with $H^{\prime}$ toral. By Lemma 6.4, $A_{0}$ (and, hence, $A$ ) has a subgroup isomorphic to $H / H^{\prime}$, as claimed.

\section{Examples.}

Example 6.6 (cf. [Se5, 1.5.1]). Let $G$ be a special group (see Remark 4.2). Then every finite abelian subgroup of $G$ is toral.

Indeed, let $V$ be a generically free linear representation of $G$ and let $H$ be a finite abelian subgroup of $G$. Since $G$ is special, $V$ is split, i.e., $A=\{1\}$ is a splitting group for $V$. On the other hand, since the origin of $V$ is a smooth $H$-fixed point, there exists a toral subgroup $H_{T}$ of $H$ such that $H / H_{T}$ is isomorphic to a subgroup of $A=\{1\}$. In other words, $H=H_{T}$ is toral, as claimed.

Example 6.7. Let $a_{1}, \ldots, a_{n}$ be independent variables over $k$ and let $q=$ $\left\langle a_{1}, \ldots, a_{n}\right\rangle$ be the generic quadratic form of dimension $n$. Then any splitting group of $q$ contains a copy of $(\mathbb{Z} / 2 \mathbb{Z})^{\left[\frac{n+1}{2}\right]}$. 
The proof is the same as in Proposition 5.1(b), with Theorem 1.2 used in place of Theorem 1.1.

Example 6.8. Let $G=E_{7}$ (adjoint) or $E_{8}$. Suppose $G \hookrightarrow S$, where $S=$ $\mathrm{GL}_{n}, \mathrm{SL}_{n}$ or $\mathrm{Sp}_{2 n}$ for some $n$. Then any splitting group of $S$ (viewed as a $G$-variety with respect to the left multiplication action) contains a copy of $(\mathbb{Z} / 2 \mathbb{Z})^{2}$.

Indeed, $G$ has an elementary abelian 2 -subgroup $H$ of depth $\geq 2$; see Propositions 5.3 and 5.7. If $X=\bar{S}$ is a smooth projective model for $S$ (as an $S$-variety) then the argument of Corollary 4.8 shows that $H$ has a fixed point in $X$. Thus we can apply Theorem 1.2 to $X$.

\section{Abelian subgroups of $\mathrm{PGL}_{n}$.}

The rest of this paper will be devoted to applications of Theorem 1.2 (with $G=\mathrm{PGL}_{n}$ ) to the theory of central simple algebras. In this section we lay the foundation for these applications by studying finite abelian subgroups of $\mathrm{PGL}_{n}$.

Symplectic modules. We begin by recalling the notion of a symplectic module from [TA2]. Let $H$ be an abelian group; in the sequel we shall refer to such groups as $\mathbb{Z}$-modules or just modules. We will always assume $H$ is finite. A skew-symmetric form on $H$ is a skew-symmetric $\mathbb{Z}$-bilinear map $\omega: H \times H \longrightarrow \mathbb{Q} / \mathbb{Z}$. If $H$ is written multiplicatively, we will usually identify $\mathbb{Q} / \mathbb{Z}$ with the multiplicative group of roots of unity in $k^{*}$. A subgroup (or, equivalently, a $\mathbb{Z}$-submodule) $H^{\prime}$ of $H$ is called isotropic if $\omega\left(h, h^{\prime}\right)=0$ for every $h, h^{\prime} \in H^{\prime}$.

We will say that $\omega$ is symplectic if it is nondegenerate, i.e., the homomorphism $H \longrightarrow H^{*}$ it defines, is an isomorphism. If $\omega$ is symplectic then a subgroup $H^{\prime} \subset H$ is called Lagrangian if it is a maximal isotropic subgroup, i.e., if $H^{\prime}$ is not contained in any other isotropic subgroup. It is easy to see that $H^{\prime}$ is Lagrangian if and only if it is isotropic and $\left|H^{\prime}\right|^{2}=|H|$; cf. [TA2, Corollary 3.1].

Lemma 7.1. Let $(H, \omega)$ be a symplectic module of order $n^{2}$.

(a) If $\Lambda$ is a Lagrangian submodule then $H / \Lambda \simeq \Lambda$ (as abelian groups).

(b) Let $H_{1}$ be a subgroup of $H$, and let $I$ be an isotropic subgroup of $H_{1}$. Then $H_{1} / I$ contains an isomorphic copy of $I_{1}$, where $I_{1}$ is an isotropic subgroup of $H$ and $\left|H_{1}\right|$ divides $n\left|I_{1}\right|$.

Proof. (a) For $h \in H$, let $\chi_{h}: \Lambda \longrightarrow k^{*}$ be the character given by $\chi_{h}(l)=$ $\omega(h, l)$. Then $h \mapsto \chi_{h}$ is a group homomorphism $\phi: H \longrightarrow \Lambda^{*}$. Since $\omega$ is nondegenerate, $\phi$ is onto. Since $\Lambda$ is Lagrangian, $\operatorname{Ker}(\phi)=\Lambda$. Thus $H / \Lambda \simeq \Lambda^{*}$. Since $\Lambda^{*} \simeq \Lambda$, the lemma follows.

(b) We may assume without loss of generality that $I$ is a maximal isotropic subgroup of $H_{1}$. Indeed, let $I_{\max }$ be a maximal isotropic subgroup of $H_{1}$ 
containing $I$. Suppose we can find an isotropic subgroup $I_{1}$ such that $\left|H_{1}\right|$ divides $n\left|I_{1}\right|$ and $H / I_{\max }$ has a subgroup isomorphic to $I_{1}$. Since $H / I_{\max }$ is isomorphic to a quotient, and hence a subgroup of $H / I$ (see Lemma 6.4), the same $I_{1}$ will work for $I$.

Thus we may (and will) assume that $I$ is a maximal isotropic subgroup of $H_{1}$. Let $\Lambda$ be a Lagrangian subgroup of $H$ containing $I$. Then $\Lambda \cap H_{1}=I$ and thus $H_{1} / I \hookrightarrow H / \Lambda \simeq \Lambda$; the last isomorphism is given by part (a). Denote the image of $H_{1} / I$ in $\Lambda$ by $I_{1}$. Then

$$
\left|H_{1}\right|=|I| \cdot\left|H_{1} / I\right|=|I| \cdot\left|I_{1}\right|
$$

divides $|\Lambda| \cdot\left|I_{1}\right|=n\left|I_{1}\right|$, as claimed.

Definition 7.2 (cf. [TA2, Section 4]). Let $A$ be an abelian group. We define a skew-symmetric form $\omega_{A}$ on $A \times A^{*}$ by

$$
\omega_{1}\left(a_{1} \oplus \chi_{1}, a_{2} \oplus \chi_{2}\right)=\chi_{1}\left(a_{2}\right)-\chi_{2}\left(a_{1}\right) .
$$

Lemma 7.3. (a) $\left(A \times A^{*}, \omega_{A}\right)$ is a symplectic module, and $A \times\{1\}$ is a Lagrangian submodule.

(b) Moreover, every symplectic module $H$ is of the form $\left(A \times A^{*}, \omega_{A}\right)$ for a suitable Lagrangian submodule $A$ of $H$.

(c) Let $(H, \omega)$ be a symplectic module, $H \simeq(\mathbb{Z} / p \mathbb{Z})^{2 r}$. If $s \leq r$ then $(H, \omega)$ has a symplectic submodule $\left(H_{1}, \omega_{\mid H_{1}}\right)$ of rank $2 \mathrm{~s}$.

Proof. Parts (a) and (b) are proved in [TA2, Section 4]. Proof of (c): By part (b), we can write $(H, \omega)$ as $\left(A \times A^{*}, \omega_{A}\right)$, where $A=(\mathbb{Z} / p \mathbb{Z})^{r}$. Let $e_{1}, \ldots, e_{r}$ be an $\mathbb{Z} / p \mathbb{Z}$-basis of $A$, viewed as an $r$-dimensional vector space over $\mathbb{Z} / p \mathbb{Z}$. Then the module $H_{1}$ spanned by $\left(e_{i}, 1\right)$ and $\left(1, e_{j}^{*}\right)$ as $i, j$ range from 1 to $s$, has the desired properties.

The form $\alpha_{H}$. Abelian subgroups of $\mathrm{PGL}_{n}$ are naturally endowed with a skew-symmetric bilinear form.

Definition 7.4. Let $H$ be a finite abelian subgroup of $\mathrm{PGL}_{n}$. For $a, b \in$ $H$ define $\alpha_{H}(a, b)=A B A^{-1} B^{-1}$, where $A$ and $B$ are elements of $\mathrm{GL}_{n}$ representing $a$ and $b$ respectively. It is easy to see that $\alpha_{H}(a, b)$ does not depend of the choice of $A$ and $B$ and $\alpha_{H}: H \times H \longrightarrow k^{*}$ defined this way, is a skew-symmetric form on $H$.

Lemma 7.5. Let $H$ be a finite abelian subgroup of $\mathrm{PGL}_{n}$. Then the following conditions are equivalent.

(a) $H$ lifts to an abelian subgroup of $\mathrm{SL}_{n}$.

(b) $H$ is toral.

(c) The skew-symmetric form $\alpha_{H}: H \times H \longrightarrow k^{*}$ given in Definition 7.4 is trivial, i.e., $\alpha_{H}(a, b)=1$ for every $a, b \in H$. 
Proof. (a) $\Longrightarrow$ (b). Recall that every finite abelian subgroup of $\mathrm{SL}_{n}$ can be simultaneously diagonalized and hence, is toral. (Alternatively, since $\mathrm{SL}_{n}$ is a special group, this follows from Example 6.6.) The tori of $\mathrm{PGL}_{n}$ are precisely the images of the tori in $\mathrm{SL}_{n}$ under the natural projection $\mathrm{SL}_{n} \longrightarrow \mathrm{PGL}_{n}$, and part (b) follows.

(b) $\Longrightarrow$ (c). Suppose $H$ is contained in a maximal torus $T \subset \mathrm{PGL}_{n}$ and let $S$ be the preimage of $T$ in $\mathrm{SL}_{n}$. Then $S$ is a maximal torus of $\mathrm{SL}_{n}$. Thus any $a, b \in H$ can be lifted to, respectively, $A, B \in S$. Since $A$ and $B$ commute, we conclude that $\alpha_{H}(a, b)=A B A^{-1} B^{-1}=1$, as claimed.

(c) $\Longrightarrow$ (a). Since $\alpha_{H}$ is trivial, the preimage of $H$ in $\mathrm{SL}_{n}$ is a finite abelian group.

The embedding $\phi$. We will now show that any symplectic module $H$ can be obtained from an abelian subgroup of $\mathrm{PGL}_{n}$, as above, with $n=\sqrt{|H|}$. Note that by Lemma 7.3(b) we may assume $H=\left(A \times A^{*}, \omega_{A}\right)$ for some abelian group $A$.

Definition 7.6 (cf. [RY, Definitions 8.7 and 8.10]). Let $A$ is an abelian group of order $n$. We define the embedding

$$
\phi: A \times A^{*} \hookrightarrow \mathrm{PGL}_{n}
$$

as follows. Identify $\mathrm{PGL}_{n}$ with $\operatorname{PGL}(V)$, where $V=k[A]=$ the group algebra of $A$. The group $A$ acts on $V$ by the regular representation $a \mapsto$ $P_{a} \in \mathrm{GL}(V)$, where

$$
P_{a}\left(\sum_{b \in A} c_{b} b\right)=\sum_{b \in A} c_{b} a b
$$

for any $a \in A^{*}$ and $c_{b} \in k$. The dual group $A^{*}$ acts on $V$ by the representation $\chi \mapsto D_{\chi} \in \mathrm{GL}(V)$, where

$$
D_{\chi}\left(\sum_{a \in A} c_{a} a\right)=\sum_{a \in A} c_{a} \chi(a) a
$$

for any $\chi \in A^{*}$ and $c_{a} \in k$. We define $\phi$ by

$$
\phi(a, \chi)=\text { the image of } P_{a} D_{\chi} \text { in } \operatorname{PGL}(V) .
$$

Lemma 7.7. Let $A$ be a finite abelian group, $a, b \in A$ and $\chi, \mu \in A^{*}$. Then:

(a) $D_{\chi} P_{a}=\chi(a) P_{a} D_{\chi}$.

(b) $\left(P_{a} D_{\chi}\right)\left(P_{b} D_{\mu}\right)\left(P_{a} D_{\chi}\right)^{-1}=\chi(b) \mu^{-1}(a)\left(P_{b} D_{\mu}\right)$.

(c) The embedding $\phi$ of Definition 7.6 is a monomorphism of groups, and $\phi\left(A \times A^{*}\right)$ is subgroup of $\mathrm{PGL}_{n}$ isomorphic to $A \times A^{*}$.

Proof. See [RY, Lemmas 8.8 and 8.11(i)]. 
Lemma 7.8. Let $A$ be an abelian group of order $n$ and let $\phi: A \times A^{*} \hookrightarrow$ $\mathrm{PGL}_{n}$ be the embedding of Definition 7.6. Then $\phi$ induces an isomorphism of $\left(A \times A^{*}, \omega_{A}\right)$ and $\left(\phi\left(A \times A^{*}\right), \alpha\right)$ as modules with skew-symmetric forms, where $\omega_{A}$ is as in Definition 7.2 and $\alpha=\alpha_{\phi\left(A \times A^{*}\right)}$ is as in Definition 7.4. In particular, $\left(\phi\left(A \times A^{*}\right), \alpha\right)$ is a symplectic module.

Proof. Let $h_{1}=\left(a_{1}, \chi_{1}\right)$ and $h_{2}=\left(a_{2}, \chi_{2}\right) \in A \times A^{*}$. Then we want to show that

$$
\alpha\left(\phi\left(h_{1}\right), \phi\left(h_{2}\right)\right)=\omega_{A}\left(h_{1}, h_{2}\right)=\chi_{1}\left(a_{2}\right) \chi_{2}\left(a_{1}\right)^{-1} .
$$

On the other hand, by definition of $\alpha$, we have

$$
\alpha\left(\phi\left(h_{1}\right), \phi\left(h_{2}\right)\right)=\left(P_{a_{1}} D_{\chi_{1}}\right)\left(P_{a_{2}} D_{\chi_{2}}\right)\left(P_{a_{1}} D_{\chi_{1}}\right)^{-1}\left(P_{a_{2}} D_{\chi_{2}}\right)^{-1} .
$$

The desired equality now follows from Lemma 7.7(b).

Corollary 7.9. Let $A$ be an abelian group of order $n=p^{r}$. Then the subgroup $H=\phi\left(A \times A^{*}\right) \subset \mathrm{PGL}_{n}$ is of depth $r$.

Proof. Let $H_{T}$ be any maximal (with respect to inclusion) toral subgroup of $H$. By Lemma 7.5, $H_{T}$ is isotropic; as it is maximal, it is Lagrangian. The index $\left[H: H_{T}\right]=n^{2} / n=n=p^{r}$, and hence, the depth of $H$ is $r$.

If $n=p^{r}$ then the depth of any $p$-subgroup of $\mathrm{PGL}_{n}$ is $\leq r$. This can be shown directly or, alternatively, derived from Theorem 1.1, since any central simple algebra of degree $n$ is split by a degree $n$ extension of its center.

\section{Symplectic modules and division algebras.}

We are now ready to proceed with our results on division algebras.

When is $\operatorname{RMaps}_{\mathrm{PGL}_{n}}\left(X, \mathrm{M}_{n}\right)$ a division algebra? We begin with an application of Theorem 1.1.

Let $X$ be a generically free irreducible $\mathrm{PGL}_{n}$-variety. Recall that $A=$ $\operatorname{RMaps}_{\mathrm{PGL}_{n}}\left(X, \mathrm{M}_{n}\right)$ is a central simple algebra with the center $Z(A)=$ $k(X)^{\mathrm{PGL}_{n}} ; A$ is of the form $\mathrm{M}_{s}(D)$, where $D$ is a division algebra. The degree $d$ of $D$ is called the index of $A$, and $s d=n$. The following lemma relates smooth points in $X$ fixed by finite abelian subgroups of $\mathrm{PGL}_{n}$, to the index of $A$.

Let $H$ be a finite abelian subgroup of $\mathrm{PGL}_{n}$. The skew-symmetric form $\alpha_{H}$ on $H$ may be singular; the quotient $H / \operatorname{Ker}\left(\alpha_{H}\right)$ is a symplectic module, and hence, $\left|H / \operatorname{Ker}\left(\alpha_{H}\right)\right|=m^{2}$ for some integer $m$.

Lemma 8.1. With the notations as above, suppose that $H$ has a smooth fixed point $x \in X$.

Then the index of $A$ is divisible by $m$. In particular, $m \mid n$, and if $H=$ $\phi_{P}\left(P \times P^{*}\right)$ where $P$ is an abelian group of order $n($ so that $m=n)$, then $A$ is a division algebra. 
Proof. Let $F$ be the center of $D$ (and of $A=\mathrm{M}_{s}(D)$ ), and let $K$ be a maximal subfield of $D$. Recall that $[K: F]=d=\operatorname{deg}(D)=\operatorname{index}(A)$ and that $K$ is a splitting field of $A$. By Theorem $1.1, d$ is divisible by $\left|H / H_{T}\right|$ where $H_{T}$ is some toral subgroup of $H$. By Lemma 7.5, $H_{T}$ is isotropic in $H$; we may assume that $H_{T}$ is a maximal isotropic subgroup of $H$. Then $H_{T} \supset \operatorname{Ker}\left(\alpha_{H}\right)$, and the image of $H_{T}$ in $H / \operatorname{Ker}\left(\alpha_{H}\right)$ is Lagrangian; it follows that $\left|H / H_{T}\right|=m$, i.e., $d$ is divisible by $m$.

The equality $s d=n$ implies then that $m \mid n$, and if $m=n$ then $s=1$, i.e., $A$ is a division algebra.

Proof of Theorem 1.3. The following proposition is an application of Theorem 1.2.

Proposition 8.2. Let $H$ be a finite abelian subgroup of $\mathrm{PGL}_{n}$ of order $n^{2}=p^{2 r}$, such that $\left(H, \alpha_{H}\right)$ is a symplectic module (i.e., $\alpha_{H}$ is nondegenerate on $H$; see Definition 7.4). Suppose $X^{\prime} \rightarrow X$ is a rational cover of irreducible generically free $\mathrm{PGL}_{n}$-varieties, $p^{e}$ is the largest power of $p$ dividing $\operatorname{deg}\left(X^{\prime} / X\right)$, and $X$ has a smooth point fixed by $H$. Then any splitting group $A^{\prime}$ for $X^{\prime}$ contains an isomorphic copy of some isotropic subgroup $I_{1} \subset H$, where $\left|I_{1}\right| \geq p^{r-e}$.

In particular, if $e=0, A^{\prime}$ contains an isomorphic copy of a Lagrangian subgroup of $H$.

Proof. By the Going Up Theorem 2.7(a), $X^{\prime}$ has an $H_{1}$-fixed point for some subgroup $H_{1}$ of $H$ of order $p^{2 r-e}$. By Theorem 1.2, $A^{\prime}$ contains a copy of $H_{1} / I$, where $I$ is a toral subgroup of $H_{1}$. Lemma 7.5 says that $\alpha_{H}$ restricted to $I$ is trivial, i.e., $I$ is an isotropic subgroup. Thus by Lemma 7.1(b), $H_{1} / I$ contains a copy of $I_{1}$, where $I_{1}$ is an isotropic subgroup of $H_{1}$ and $\left|H_{1}\right|$ divides $p^{r} \cdot\left|I_{1}\right|$. Since $\left|H_{1}\right| \geq p^{2 r-e}$, this translates into $\left|I_{1}\right| \geq p^{r-e}$, as claimed.

We now continue with the proof of Theorem 1.3. Recall that $\operatorname{UD}(n)=$ $\operatorname{RMaps}_{\mathrm{PGL}_{n}}\left(X, \mathrm{M}_{n}\right)$, where $X=\mathrm{M}_{n} \times \mathrm{M}_{n}$, with $\mathrm{PGL}_{n}$ acting by simultaneous conjugation; see Example 3.1. Let $D=\mathrm{UD}(n) \otimes_{Z(n)} K$, as in the statement of Theorem 1.3. Then $D=\operatorname{RMaps}_{\mathrm{PGL}_{n}}\left(X_{K}, \mathrm{M}_{n}\right)$. Recall that we are assuming $n=p^{r}$, and $p^{e}$ is the highest power of $p$ dividing $[K: Z(n)]=\operatorname{deg}\left(X_{K} / X\right)$. Also recall that $A$ is a splitting group for $D$ if and only if $A$ is a splitting group for $X_{K}$ (as a $\mathrm{PGL}_{n}$-variety); see Definition 6.1 .

Note that $X=\mathrm{M}_{n} \times \mathrm{M}_{n}$ has a smooth point (namely, the origin) fixed by all of $G$. Let $P$ be an abelian $p$-group of order $n=p^{r}$; then $H=$ $\phi_{P}\left(P \times P^{*}\right)$ is an abelian $p$-subgroup of $\mathrm{PGL}_{n}$. By Lemma 7.8, $\left(H, \alpha_{H}\right)$ is a symplectic module. Applying Proposition 8.2 to $H$ and remembering that every symplectic module of order $p^{2 r}$ is isomorphic to one of the form $\phi_{P}\left(P \times P^{*}\right)$ for some $P$ (see Lemmas 7.3(b) and 7.8), we obtain the following generalization of [TA1, Corollary 7.2] (in characteristic 0): 
Proposition 8.3. Let $Z\left(p^{r}\right)$ be the center of the generic division algebra $\mathrm{UD}\left(p^{r}\right)$, let $K$ be a field extension of $Z\left(p^{r}\right)$ and let $D=\mathrm{UD}\left(p^{r}\right) \otimes_{Z\left(p^{r}\right)} K$. Suppose $p^{e}$ is the highest power of $p$ dividing $\left[K: Z\left(p^{r}\right)\right]$, where $e$ is a nonnegative integer and $e \leq r-1$.

If $A$ is a splitting group of $D$ then for every symplectic module $H$ of order $p^{2 r}$, there exists an isotropic submodule $I_{1}$ of order $p^{r-e}$ such that $A$ contains an isomorphic copy of $I_{1}$.

In order to finish the proof of Theorem 1.3 we use a comparison argument, as in the proof of [TA1, Theorem 7.3]. Let

$$
H_{1}=\phi_{P_{1}}\left(P_{1} \times P_{1}^{*}\right) \text { and } H_{2}=\phi_{P_{2}}\left(P_{2} \times P_{2}^{*}\right),
$$

where

$$
P_{1}=(\mathbb{Z} / p \mathbb{Z})^{r} \text { and } P_{2}=\mathbb{Z} / p^{r} \mathbb{Z}
$$

By Proposition 8.3, $A$ contains an isomorphic copy $I_{1}$ of an isotropic subgroup of $H_{1}$, and an isomorphic copy $I_{2}$ of an isotropic subgroup of $H_{2}$, such that $\left|I_{1}\right|=\left|I_{2}\right|=p^{r-e}$. Since $H_{1} \simeq(\mathbb{Z} / p \mathbb{Z})^{2 r}$ and $H_{2} \simeq\left(\mathbb{Z} / p^{r} \mathbb{Z}\right)^{2}$, $I_{1} \simeq(\mathbb{Z} / p \mathbb{Z})^{r-e}$ and $I_{2}$ has rank $\leq 2$. We may assume without loss of generality that both $I_{1}$ and $I_{2}$ are contained in the same Sylow $p$-subgroup $A_{p}$ of $A$. Since the intersection of $I_{1}$ and $I_{2}$ has exponent $p$ and rank $\leq 2$, we see that

$$
\left|A_{p}\right| \geq\left|I_{1} I_{2}\right|=\frac{\left|I_{1}\right|\left|I_{2}\right|}{\left|I_{1} \cap I_{2}\right|} \geq \frac{p^{2 r-2 e}}{p^{2}}=p^{2 r-2 e-2},
$$

where $I_{1} I_{2}=\left\{\gamma_{1} \gamma_{2} \mid \gamma_{1} \in I_{1}, \gamma_{2} \in I_{2}\right\}$.

This shows that $\left|A_{p}\right|$ is divisible by $p^{2 r-2 e-2}$ and, hence, so is $|A|$, as claimed.

Remark 8.4. The only property of $X=\mathrm{M}_{n} \times \mathrm{M}_{n}$ used in the above proof is that each of the finite abelian subgroups $H_{1} \simeq\left(\mathbb{Z} / p^{r} \mathbb{Z}\right)^{2}$ and $H_{2} \simeq(\mathbb{Z} / p \mathbb{Z})^{r}$ of $\mathrm{PGL}_{n}$ has a smooth fixed point in $X$. Thus our argument shows that Theorem 1.3 remains valid if the universal division algebra $\operatorname{UD}(n)$ is replaced by the algebra $U^{\prime}=\operatorname{RMaps}_{\mathrm{PGL}_{n}}\left(X, \mathrm{M}_{n}\right)$, where $X$ is an irreducible generically free $\mathrm{PGL}_{n}$-variety $X$ such that $H_{i}$ has a smooth fixed point in $X$ for $i=1,2$. There are many choices for such $X$; in particular, by Proposition $8.6 X$ can be chosen so that $\operatorname{dim}\left(X / \mathrm{PGL}_{n}\right)=2 r$ or, equivalently, $\operatorname{trdeg}_{k}\left(Z\left(U^{\prime}\right)\right)=2 r$, where $Z\left(U^{\prime}\right)$ is the center of $U^{\prime}$.

Remark 8.5. Tignol and Amitsur showed that if $A$ is an abelian splitting group of $\mathrm{UD}\left(p^{r}\right)$ and

$$
A_{p} \cong \mathbb{Z} / p^{n_{1}} \mathbb{Z} \times \cdots \times \mathbb{Z} / p^{n_{l}} \mathbb{Z}
$$

is its Sylow $p$-subgroup then $n_{\nu}+n_{\nu+1} \geq[r / \nu]$ for every $\nu=1,2, \ldots$; see [TA1, Theorem 7.4]. Consequently, the order of $A_{p}$ (and, hence, of $A$ ) 
is divisible by $p^{f(r)}$, where

$$
f(r)=r+\sum_{\nu \geq 3}\left\{\frac{[r / \nu]}{2}\right\} ;
$$

see [TA1, Theorem 7.5]. Here $[x]$ is the greatest integer $\leq x$ and $\{x\}$ is the smallest nonnegative integer $\geq x$. Note that $f(r)=\frac{1}{2} r \ln (r)+O(r)$, as $r \rightarrow \infty$; see [TA1, Remark 7.5] (a more precise asymptotic estimate is given in [TA2, Corollary 6.2]).

The same assertions hold if $A$ is an abelian splitting group for any primeto- $p$ extension of $\mathrm{UD}\left(p^{r}\right)$ : The proof given in [TA1, Theorem 7.5] goes through unchanged, except that we use Proposition 8.3 (with $e=0$ ) in place of [TA1, Corollary 7.2],

Moreover, let $D=\mathrm{UD}\left(p^{r}\right) \otimes_{Z\left(p^{r}\right)} K$, where $p^{e}$ is the highest power of $p$ which divides $\left[K: Z\left(p^{r}\right)\right]$, as in the statement of Theorem 1.3. Suppose $A$ is a splitting group of $D$ and $A_{p}$ is the Sylow $p$-subgroup of $A$. If $A_{p}$ is as in (8.3) then a slight modification of the proof of [TA2, Lemma 6.1] (again, based on Proposition 8.3) shows that $n_{\nu}+n_{\nu+1} \geq[r / \nu]-e$ for every $\nu=1,2, \ldots$ and consequently, the order of $A_{p}$ (and, hence, of $A$ ) is divisible by $p^{f_{e}(r)}$, where

$$
f_{e}(r)=r-e+\sum_{\nu \geq 3}\left\{\frac{[r / \nu]-e}{2}\right\} .
$$

It is easy to see that for a fixed $e$ and large $r, f_{e}(r)$ also grows as $\frac{1}{2} r \ln (r)+$ $O(r)$.

Reduction of Theorem 1.4 to a geometric problem. Our proof of Theorem 1.4 will be based on Proposition 8.2. The idea is to construct a

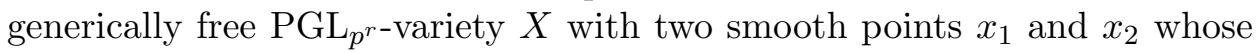
stabilizers contain "incompatible" symplectic modules $H_{1}$ and $H_{2}$. Let $P_{1}$ and $P_{2}$ be as in (8.2); this time we take

$$
H_{2}=\phi_{P_{2}}\left(P_{2} \times P_{2}^{*}\right) \simeq\left(\mathbb{Z} / p^{r} \mathbb{Z}\right)^{2},
$$

as in (8.1), but allow $H_{1}$ to be smaller:

$$
H_{1}=\operatorname{rank} 6 \text { symplectic subgroup of } \phi_{P_{1}}\left(P_{1} \times P_{1}^{*}\right) \text {. }
$$

Note that $H_{1} \simeq(\mathbb{Z} / p \mathbb{Z})^{6}$ with desired properties exists by Lemma 7.3(c).

Suppose $X$ is an irreducible generically free $\mathrm{PGL}_{n}$-variety, and $x_{1}, x_{2}$ are smooth points of $X$ such that $x_{i}$ is fixed by $H_{i}$. Let $D$ be the algebra $\operatorname{RMaps}_{\mathrm{PGL}_{n}}\left(X, \mathrm{M}_{n}\right)$. Since $X$ has a smooth point fixed by $H_{2}$, Lemma 8.1 tells us that $D$ is a division algebra. Moreover, in view of Proposition 8.2 (with $X=X^{\prime}$ ) any splitting group $A$ of $X$ (or equivalently, of $D$ ) will contain subgroups $L_{1}$ and $L_{2}$ which are isomorphic to Lagrangian submodules of $H_{1}$ and $H_{2}$, respectively. Note that $L_{1} \simeq(\mathbb{Z} / p \mathbb{Z})^{3}$ and $L_{2} \simeq\left(\mathbb{Z} / p^{i} \mathbb{Z}\right) \times$ 
$\left(\mathbb{Z} / p^{r-i} \mathbb{Z}\right)$, for some $0 \leq i \leq r$. Then $L_{1} \cap L_{2}$ is an abelian group of exponent $p$ and rank $\leq 2$. Thus

$$
|A| \geq\left|L_{1} L_{2}\right|=\frac{\left|L_{1}\right|\left|L_{2}\right|}{\left|L_{1} \cap L_{2}\right|} \geq \frac{p^{3} p^{r}}{p^{2}}=p^{r+1} ;
$$

this shows that $D$ is not a crossed product. The same argument shows that any prime-to- $p$ extension of $D$ is not a crossed product.

Thus, in order to prove Theorem 1.4 it is sufficient to construct an irreducible generically free $\mathrm{PGL}_{n}$-variety $X$ such that $\operatorname{trdeg}_{k} k(X)^{\mathrm{PGL}_{n}}=$ $\operatorname{dim}\left(X / \mathrm{PGL}_{n}\right)=6$ and $X$ has smooth points $x_{1}$ and $x_{2}$ such that $x_{i}$ is fixed by $H_{i}$.

Note that both $H_{1}$ and $H_{2}$ are contained in the finite subgroup $G$ of $\mathrm{PGL}_{n}$ generated by the permutation matrices and by the diagonal matrices all of whose entries are $p^{r}$ th roots of unity. We will construct $X$ as $\mathrm{PGL}_{n} *_{G} Y$, where $Y$ is a 6 -dimensional primitive $G$-variety with two points, $y_{1}$ and $y_{2}$ such that $H_{i}$ fixes $y_{i}$. Indeed, if $Y$ is as above then the points $x_{1}=$ $\left(1_{\mathrm{PGL}_{n}}, y_{1}\right)$ and $x_{2}=\left(1_{\mathrm{PGL}_{n}}, y_{2}\right)$ of $X$ have the desired properties. (Recall that $\mathrm{PGL}_{n} *_{G} Y$ is defined as the geometric quotient of $\mathrm{PGL}_{n} \times Y$ by the $G$-action given by $g \cdot(h, y)=\left(h g^{-1}, g y\right)$; see [PV, Section 4.8].)

Therefore, in order to prove Theorem 1.4 it is enough to establish the following result.

Proposition 8.6. Let $G$ be a finite group and let $H_{1}, \ldots, H_{s}$ be abelian subgroups of $G, r_{i}=\operatorname{rank}\left(H_{i}\right)$ and $r=\max \left\{r_{i} \mid i=1, \ldots, s\right\}$. Then there exists a generically free primitive $r$-dimensional projective $G$-variety $Y$ with smooth points $y_{1}, \ldots, y_{s}$ such that $H_{i} \subset \operatorname{Stab}\left(y_{i}\right)$.

Remark 8.7. Note that $\operatorname{dim}(Y)$ cannot be less than $r$. More precisely, if $H$ is a finite abelian group, $Y$ is a quasiprojective $H$-variety, and $y$ is a smooth point of $Y$ fixed by $H$ then

$$
\operatorname{codim}_{y}\left(Y^{H}\right) \geq \operatorname{rank}(H) .
$$

Indeed, assume the contrary: $\operatorname{codim}_{y}\left(Y^{H}\right)<\operatorname{rank}(H)$. By Lemma 2.1(a), $y$ has an $H$-invariant affine neighborhood in $Y$. Replacing $Y$ by this neighborhood, we may assume $Y$ is affine. By the Luna Slice Theorem $[\mathbf{P V}$, Corollary to Theorem 6.4], $Y^{H}$ is smooth at $y$ and

$$
\operatorname{dim}\left(T_{y}(Y)\right)-\operatorname{dim}\left(T_{y}(Y)^{H}\right)=\operatorname{codim}_{y}\left(Y^{H}\right)<\operatorname{rank}(H) ;
$$

hence, the action of $H$ on $T_{y}(Y)$ cannot be faithful. In other words, there exists a subgroup $H^{\prime} \subset H, H^{\prime} \neq\{1\}$, which acts trivially on $T_{y}(Y)$. Applying $\left[\mathbf{P V}\right.$, Corollary to Theorem 6.4] to the action of $H^{\prime}$ on $Y$, we see that $H^{\prime}$ acts trivially on all of $Y$. This contradicts our assumption that the $G$-action on $Y$ is generically free. 


\section{Constructing a $G$-variety with prescribed stabilizers.}

As we have just seen, Theorem 1.4 follows from Proposition 8.6. This section will thus be devoted to proving Proposition 8.6. Our general approach is to first construct a higher-dimensional variety with desired properties (this is easy), then replace it by a "generic" $G$-invariant hypersurface passing through $y_{1}, \ldots, y_{s}$, thus reducing the dimension by 1 . To carry out this program, we first reduce to a situation where $Y^{H_{i}}$ has the highest possible dimension at $y_{i}$ (Theorem 9.3), then apply Theorem 9.7, which may be viewed as a weak form of Bertini's theorem in the equivariant setting.

A local system of parameters. The following lemma summarizes some known facts about the local geometry of a smooth $G$-variety near a point fixed by a finite abelian group.

Lemma 9.1. Let $H$ be a finite abelian group, let $X$ be a smooth quasiprojective $H$-variety, and let $D_{1}, \ldots, D_{l}$ be $H$-invariant hypersurfaces passing through a point $x \in X$ and intersecting transversely at $x$.

(1) There exists a local coordinate system (a regular system of parameters) $u_{1}, \ldots, u_{n}$ with the following properties:

(i) The group $H$ acts on each $u_{i}$ by a character $\xi_{i}$;

(ii) $u_{i}$ is the local equation of $D_{i}$ for $i=1, \ldots, l$;

(iii) The germ of the fixed-point set $X^{H}$ at $x$ is given by the local equations $u_{i_{1}}=\cdots=u_{i_{t}}=0$ where $\left\{i_{1}, \ldots, i_{t}\right\}$ is the set of all subscripts $i$ for which the character $\xi_{i}$ is nontrivial.

(2) Let $\pi: X^{\prime} \longrightarrow X$ be the blowup with the center $Z$ given by the local equations $u_{j_{1}}=\cdots=u_{j_{s}}=0$ at $x$. (In particular, we can take $Z=X^{H}$.) Let $\widetilde{D}_{i} \subset X^{\prime}$ be the strict transform of $D_{i}$. Then we have:

(i) $\widetilde{D}_{1}, \ldots, \widetilde{D}_{l}$ and the exceptional divisor $\pi^{-1}(Z)$ are in normal crossing in a neighborhood of $\pi^{-1}(x)$;

(ii) the natural isomorphism $\pi^{-1}(x) \cong \mathbb{P}\left(T_{x} X / T_{x} Z\right)$ identifies $\widetilde{D}_{i} \cap$ $\pi^{-1}(x)$ with $\mathbb{P}\left(L_{i}\right)$, where $L_{i}$ is an $H$-invariant subspace of $T_{x} X / T_{x} Z$ of codimension 0 or 1 .

Note that if $u_{i}$ is a local equation of $D_{i}$ and $H$ acts on $u_{i}$ by a character $\xi_{i}$ then $H$ acts by the character $\xi_{i}$ on the conormal space $\left(T_{x} X / T_{x} D_{i}\right)^{*}$.

Proof. By Lemma 2.1(a), we may assume without loss of generality that $X$ is affine.

(1) Denote by $\mathcal{O}_{x}$ the local ring of $X$ at $x$, by $\mathfrak{m}_{x}$ its maximal ideal, and by $\mathfrak{p}_{D_{i}}$ the ideal of $D_{i}$ in $\mathcal{O}_{x}$.

To construct $u_{1}, \ldots, u_{l}$, note that the group $H$ acts on $\mathcal{O}_{x}$, the ideals $\mathfrak{m}_{x}$ and $\mathfrak{p}_{D_{i}}$ are $H$-invariant subspaces in $\mathcal{O}_{x}$, and the $H$-representation $\left(\mathfrak{p}_{D_{i}}+\right.$ $\left.\mathfrak{m}_{x}^{2}\right) / \mathfrak{m}_{x}^{2} \cong \mathfrak{p}_{D_{i}} /\left(\mathfrak{p}_{D_{i}} \cap \mathfrak{m}_{x}^{2}\right)$ is one-dimensional; let $\xi_{i}$ be the corresponding character of $H$. The $H$-linear epimorphism $\mathfrak{p}_{D_{i}} \longrightarrow \mathfrak{p}_{D_{i}} /\left(\mathfrak{p}_{D_{i}} \cap \mathfrak{m}_{x}\right)$ splits; 
this yields a generator $u_{i} \in \mathfrak{p}_{D_{i}}$ - a local equation of $D_{i}$ - on which $H$ acts by the character $\xi_{i}$.

To construct $u_{l+1}, \ldots, u_{n}$, consider the $H$-linear epimorphism

$$
\mathfrak{m}_{x} \longrightarrow \mathfrak{m}_{x} /\left(\mathfrak{p}_{D_{1}}+\cdots+\mathfrak{p}_{D_{l}}+\mathfrak{m}_{x}^{2}\right) \stackrel{\simeq}{=} \frac{\mathfrak{m}_{x} / \mathfrak{m}_{x}^{2}}{\sum_{i=1}^{l}\left(\mathfrak{p}_{D_{i}}+\mathfrak{m}_{x}^{2}\right) / \mathfrak{m}_{x}^{2}} ;
$$

its splitting yields the elements $u_{l+1}, \ldots, u_{n} \in \mathfrak{m}_{x}$ such that $H$ acts on each of them by a character and the images of $u_{1}, \ldots, u_{n}$ in $\mathfrak{m}_{x} / \mathfrak{m}_{x}^{2}$ form a basis there. It follows that $u_{1}, \ldots, u_{n}$ form a regular system of parameters at $x$ that satisfies properties (1)(i) and (1)(ii).

According to the Luna Slice Theorem [PV, Corollary to Theorem 6.4], $X^{H}$ is given in a neighborhood of $x$ by the local equations $u_{i_{1}}=\cdots=u_{i_{t}}=$ 0 , where $\left\{i_{1}, \ldots, i_{t}\right\}$ is the set of all subscripts $i$ for which the character $\xi_{i}$ is nontrivial. This proves part (1)(iii).

(2) Let $U$ be a small enough affine neighborhood of $x$ in $X$ so that $u_{1}, \ldots, u_{n}$ form a local coordinate system (i.e., their differentials are linearly independent) everywhere on $U$. The blown-up variety $X^{\prime}$ in a neighborhood of $\pi^{-1}(x)$ is covered by the charts $U_{i}, 1 \leq i \leq s$, where $U_{i}$ is the complement in $\pi^{-1}(U)$ of the strict transform of the subvariety $u_{j_{i}}=0$; the local coordinates in $U_{i}$ are $v_{j_{i}}=\pi^{*} u_{j_{i}}, v_{j_{i^{\prime}}}=\pi^{*} u_{j_{i^{\prime}}} / \pi^{*} u_{j_{i}}$ for $i^{\prime} \neq i$, and $v_{j}=\pi^{*} u_{j}$ for $j \notin\left\{j_{1}, \ldots, j_{s}\right\}$. The exceptional divisor in $U_{i}$ is given by the local equation $v_{j_{i}}=0$, and $\widetilde{D}_{j}$ is given by the local equation $v_{j}=0$ in case $j \neq j_{i}$, and is empty in case $j=j_{i}$. Since the local equations of $\widetilde{D}_{1}, \ldots, \widetilde{D}_{l}$ and of the exceptional divisor are elements of the same local coordinate system, they are transverse; this proves (2)(i).

The local equations in $U_{i}$ of the preimage $\pi^{-1}(x)$ are $v_{j}=0$ for $j \notin$ $\left\{j_{1}, \ldots, j_{s}\right\}$, and $v_{j_{i}}=0$; hence, $\pi^{-1}(x)$ is contained in $\widetilde{D}_{j}$ if $j \notin\left\{j_{1}, \ldots, j_{s}\right\}$, so that in this case $\widetilde{D}_{j} \cap \pi^{-1}(x)=\pi^{-1}(x)$ as claimed in (2)(ii). If $j \in$ $\left\{j_{1}, \ldots, j_{s}\right\}$ then $D_{i} \supset Z$ and $\widetilde{D}_{j} \cap \pi^{-1}(x)$ can be identified with $\mathbb{P}\left(T_{x} D_{i} / T_{x} Z\right)$; here $L_{i}=T_{x} D_{i} / T_{x} Z$ is an $H$-invariant subspace of $T_{x} X / T_{x} Z$ of codimension 1 as claimed. This completes the proof of (2)(ii).

Lemma 9.2. Let $G$ be a finite group, $H$ be an abelian subgroup of $G, X$ be a smooth quasiprojective $G$-variety and $x \in X^{H}$. Then there exists a sequence of blowups

$$
f: X_{i} \longrightarrow \ldots \longrightarrow X_{0}=X
$$

with smooth $G$-invariant centers, a point $y \in X_{i}^{H}$ satisfying $f(y)=x$, and smooth $G$-invariant hypersurfaces $D_{1}, \ldots, D_{l}$ meeting transversely, such that locally at $y$, the fixed point set $X_{i}^{H}$ coincides with $D_{1} \cap \cdots \cap D_{l}$.

Proof. By Theorem 2.5, we may assume that $\operatorname{Stab}(x)$ is commutative.

The statement of the Lemma follows from the case $H=\operatorname{Stab}(x)$. Indeed, if $X_{i}^{\operatorname{Stab}(y)}=D_{1} \cap \cdots \cap D_{l}$ locally at $y$, then by Luna's slice theorem (see 
[PV, Theorem 6.4]), $X_{i}^{H}$ is the intersection of those of $D_{j}$ for which the action of $H$ on the normal space $T_{y}\left(X_{i}\right) / T_{y}\left(D_{j}\right)$ is nontrivial; thus, $X_{i}^{H}$ is the intersection of some $D_{j}$, as claimed. (Note that the Luna Slice Theorem can be applied to the $G$-action on $X_{i}$ because $X_{i}$ is quasiprojective and, hence, every point of $X_{i}$ has an open affine $G$-invariant neighborhood; see Lemma 2.1.)

From now on we assume that $\operatorname{Stab}(x)=H$. Let $X^{\prime}=X-G \cdot \bigcup_{H^{\prime} \supset H} X^{H^{\prime}}$; this is a $G$-invariant open dense quasiprojective subvariety of $X$ containing $x$ and not containing any point whose stabilizer is strictly larger than $H$. If we can find a sequence of blowups (9.1) for $X^{\prime}$, then we can extend it to a similar sequence for $X$ by extending each blowup center in $X^{\prime}$ to its closure in $X$ and equivariantly resolving it before blowing it up, in case it is not smooth; for equivariant resolution of singularities, see either $[\mathbf{V}$, Theorem 7.6.1] or [BM, Theorem 13.2].

Thus, we may assume that $X$ does not contain points with stabilizers strictly containing $H$; this implies that the subvarieties $X^{g H g^{-1}}$ for different $g \in G$ are disjoint unless they coincide. Each of these subvarieties is smooth by Luna's slice theorem (see [PV, Corollary to Theorem 6.4]), and hence, their union $G X^{H}=\bigcup_{g \in G} X^{g H g^{-1}}$ is smooth.

Let $\operatorname{dim} X=n$, and let

$$
X_{n} \stackrel{\pi_{n}}{\longrightarrow} \ldots \stackrel{\pi_{i+1}}{\longrightarrow} X_{i} \stackrel{\pi_{i}}{\longrightarrow} \ldots \stackrel{\pi_{1}}{\longrightarrow} X_{0}=X
$$

be the sequence of blowups centered at $Z_{i}=G X_{i}^{H} \subset X_{i}$; each blowup $\pi_{i}$ is $G$-equivariant. Inductively, each $X_{i}$ has no points whose stabilizers strictly contain $H$; together with the fact that $X_{i}$ is smooth, this implies that $Z_{i}$ is smooth, and hence, $X_{i+1}$ is smooth, so that $X_{i}$ and $Z_{i}$ are smooth for every $i$.

Let $E_{i}=D_{i 1} \cup \cdots \cup D_{i i}$ be the exceptional divisor in $X_{i}$, where $D_{i j} \subset X_{i}$ is the strict transform (in $X_{i}$ ) of the exceptional divisor of $\pi_{j}: X_{j} \longrightarrow X_{j-1}$. We claim that $E_{i}$ is a normal crossing divisor. The proof is by induction on $i$. The base case, $i=0$, is obvious, since $E_{0}=$ the empty divisor, is normal crossing. For the inductive step, we assume that $E_{i}$ is a normal crossing divisor. Then $E_{i+1}$ is also a normal crossing divisor by Lemma 9.1(2)(i). This completes the proof of the claim.

To obtain the required point $y \in X_{m}^{H}$, we start with $x_{0}=x$ and inductively construct $x_{i} \in X_{i}$ satisfying $\pi_{i}\left(x_{i}\right)=x_{i-1}$ and $x_{i} \in X_{i}^{H} \cap D_{i 1} \cap \cdots \cap D_{i i}$, until we get a point $y=x_{m}$ with the desired properties.

Suppose $x_{i}$ has been constructed for some $i \geq 0$. Note that near $x_{i}$, the center $Z_{i}=G X_{i}^{H}$ coincides with $X_{i}^{H}$. 
Case 1. The germ of $X_{i}^{H}$ at $x_{i}$ does not contain the germ of $S=\bigcap_{j=1}^{i} D_{i j}$. Let

$$
W=\pi_{i+1}^{-1}\left(x_{i}\right) \cap \bigcap_{j=1}^{i+1} D_{i+1, j} \subset X_{i+1} .
$$

We claim $W \neq \emptyset$. Indeed, since $x_{i} \in X_{i}^{H}$, we have $\pi_{i+1}^{-1}\left(x_{i}\right) \subset \pi_{i+1}^{-1}\left(Z_{i}\right)=$ $D_{i+1, i+1}$, and thus

$$
W=\pi_{i+1}^{-1}\left(x_{i}\right) \cap \bigcap_{j=1}^{i} D_{i+1, j} .
$$

Since $D_{i+1, j}$ is the strict transform of $D_{i j}, \bigcap_{j=1}^{i} D_{i+1, j}$ contains the strict transform of $S=\bigcap_{j=1}^{i} D_{i j}$. Thus $W$ contains the intersection of the strict transform of $S$ with $\pi_{i+1}^{-1}\left(x_{i}\right)$. As the germ of $S$ at $x_{i}$ is not contained in the germ of the blowup center $Z_{i}$, i.e., of $X_{i}^{H}$, the strict transform of $S$ is nonempty and intersects $\pi_{i+1}^{-1}\left(x_{i}\right)$. Consequently, $W$ is nonempty, as claimed.

We now identify $\pi_{i+1}^{-1}\left(x_{i}\right)$ with $\mathbb{P}\left(T_{x_{i}} X_{i} / T_{x_{i}} Z_{i}\right)$ in the usual manner. Note that this identification is $H$-equivariant. Then by Lemma $9.1(2)(\mathrm{ii}), \pi_{i+1}^{-1}\left(x_{i}\right)$ $\cap D_{i+1, j}$ is identified with $\mathbb{P}\left(L_{j}\right)$, where $L_{j}$ is an $H$-invariant subspace of the normal space $T_{x_{i}} X_{i} / T_{x_{i}} Z_{i}$. Thus $W$ is identified with $\mathbb{P}(L)$, where $L=$ $L_{1} \cap \cdots \cap L_{i}$; see (9.2). Note that $L \neq(0)$ because $W \neq \emptyset$; moreover, $H$ acts linearly on $L$. Since $H$ is diagonalizable, it has an eigenvector in $L$, i.e., a fixed point in $W \cong \mathbb{P}(L)$. This fixed point is our new point $x_{i+1}$. By our construction, $\pi_{i+1}\left(x_{i+1}\right)=x_{i}$ and $x_{i+1} \in X_{i+1}^{H} \cap \bigcap_{j=1}^{i+1} D_{i+1, j}$, as desired.

Case 2. $X_{i}^{H}$ at $x_{i}$ contains $\bigcap_{j=1}^{i} D_{i j}$ at $x_{i}$. Note that this is necessarily the case if $i=n$. By Lemma 9.1(1), $X_{i}^{H}$ at $x_{i}$ coincides with the intersection of those of $D_{i j}$ for which the action of $H$ on $T_{x_{i}} X_{i} / T_{x_{i}} D_{i j}$ is nontrivial; in particular, $X_{i}^{H}$ at $x_{i}$ is an intersection of smooth $G$-invariant hypersurfaces meeting transversely, as required.

Thus, we see that for some $i \leq n$, Case 2 occurs and we get a point $x_{i} \in X_{i}$ with the required properties.

Resolving the action on the tangent space. In this subsection we prove the following result.

Theorem 9.3. Let $G$ be a finite group, let $X$ be a smooth quasiprojective $G$-variety, let $H_{1}, \ldots, H_{s}$ be abelian subgroups of $G$ and $x_{1}, \ldots, x_{s}$ be points of $X$ such that $x_{i}$ is fixed by $H_{i}$. Denote the rank of $H_{i}$ by $r_{i}$. Then there is a sequence of blowups $\pi: X_{m} \longrightarrow \cdots \longrightarrow X_{0}=X$ with smooth $G$-invariant centers, and points $y_{1}, \ldots, y_{s} \in X_{m}$, such that $\pi\left(y_{i}\right)=x_{i}, y_{i} \in X_{m}^{H_{i}}$, and $X_{m}^{H_{i}}$ has codimension $r_{i}$ at $y_{i}$. 
Our proof relies on the following two simple lemmas.

Lemma 9.4. Let $H$ be a finite abelian group, let $X$ be an $H$-variety and $x \in X^{H}$ be a smooth point of $X$. If $\pi: \tilde{X} \longrightarrow X$ is a blowup with a smooth $H$-invariant center $Z \subset X$ then there exists a point $\widetilde{x} \in \pi^{-1}(x)$ fixed by $H$ such that $\operatorname{dim}_{\widetilde{x}}\left(\widetilde{X}^{H}\right) \geq \operatorname{dim}_{x}\left(X^{H}\right)$.

Proof. Replacing $X$ by the set of its smooth points (which is clearly $H$ invariant), we may assume that $X$ is smooth. We claim that $\pi\left(\widetilde{X}^{H}\right)=X^{H}$. The inclusion $\pi\left(\widetilde{X}^{H}\right) \subset X^{H}$ is obvious. To prove the opposite inclusion, note that since $\pi$ is an isomorphism over $X \backslash Z$, every $y \in X^{H}-Z$ lies in $\pi\left(\widetilde{X}^{H}\right)$. On the other hand, if $y \in Z^{H}$ then $\pi^{-1}(y)$ can be identified with $\mathbb{P}\left(T_{y} X / T_{y} Z\right)$ as $H$-varieties, and the (linear) action of $H$ on $\mathbb{P}\left(T_{y} X / T_{y} Z\right)$ has a fixed point $\widetilde{y}$; then $y=\pi(\widetilde{y}) \in \pi\left(\widetilde{X}^{H}\right)$. This proves the claim, and the lemma follows.

Remark 9.5. Lemma 9.4 remains true under the more general assumption that $H$ is Levi-commutative rather than finite abelian; see Definition 2.3 and Lemma 2.4(iv). The version we stated is sufficient for our application.

Lemma 9.6. Let $A$ be a finite abelian group of rank $r$. An elementary operation on $A^{s}$ is one of the form

$$
\left(\xi_{1}, \ldots, \xi_{i}, \ldots, \xi_{s}\right) \longrightarrow\left(\xi_{1}, \ldots, \xi_{i}-\xi_{j}, \ldots, \xi_{s}\right)
$$

for some $1 \leq i, j \leq s$, where $i \neq j$.

Assume $s \geq r$. Then any $\xi=\left(\xi_{1}, \ldots, \xi_{s}\right) \in A^{s}$ can be transformed, by a finite sequence of elementary operations, into an $s$-tuple with at least $s-r$ zeros. (Here by a zero, we mean the identity element of A.)

Proof. First we note that it does no harm to permute the components of $\xi$. In other words, we may as well consider an operation of the form $\left(\xi_{1}, \ldots, \xi_{s}\right) \mapsto$ $\left(\xi_{\sigma(1)}, \ldots, \xi_{\sigma(s)}\right)$ with $\sigma \in \mathrm{S}_{n}$, as another type of elementary operation. The assertion of the lemma is then equivalent to saying that any $\xi \in A^{s}$ can be transformed, by these two types of elementary operations, into an $s$-tuple $\lambda=\left(\lambda_{1}, \ldots, \lambda_{r}, 0_{A}, \ldots, 0_{A}\right)$, where $0_{A}$ is the identity element of $A$.

We will prove this assertion by induction on $r$. Suppose $r=1$, i.e., $A=\mathbb{Z} / n \mathbb{Z}$ for some $n \geq 1$. We can use elementary operations to perform the Euclidean algorithm on $\xi_{1}$ and $\xi_{2}$. After interchanging them if necessary, we may assume $\xi_{2}=0$. (The new value of $\xi_{1}$ is the greatest common divisor of the old values of $\xi_{1}$ and $\xi_{2}$.) Applying the same procedure to $\xi_{1}$ and $\xi_{3}$, then $\xi_{1}$ and $\xi_{4}$, etc., we reduce the original $s$-tuple to $\left(\xi_{1}, 0, \ldots, 0\right)$, as claimed.

For the induction step, write $A=B \times C$, where $B$ has rank $r-1$ and $C$ is cyclic. Set $\xi_{i}=\left(\beta_{i}, \gamma_{i}\right)$, where $\beta_{i} \in B$ and $\gamma_{i} \in C$. As we saw above, after performing a sequence of elementary operations, we may assume $\gamma_{2}=\cdots=$ 
$\gamma_{s}=0_{C}$. By the induction assumption, there exists a sequence of elementary operations in $B^{s-1}$ which reduces $\left(\beta_{2}, \ldots, \beta_{s}\right)$ to $\left(\lambda_{2}, \ldots, \lambda_{r}, 0_{B}, \ldots, 0_{B}\right)$. (Note that since $r \leq s, \operatorname{rank}(B)=r-1 \leq s-1$, so that we may, indeed, use the induction assumption.) Applying the same sequence to $\left(\xi_{2}, \ldots, \xi_{s}\right)$, we reduce $\left(\xi_{1}, \ldots, \xi_{s}\right)$ to

$$
\left(\xi_{1},\left(\lambda_{2}, 0_{C}\right), \ldots,\left(\lambda_{r}, 0_{C}\right), 0_{A}, \ldots, 0_{A}\right) \in A^{s} .
$$

This completes the proof of the lemma.

Proof of Theorem 9.3. By (8.4), we have

$$
\operatorname{codim}_{x_{i}}\left(X^{H_{i}}\right) \geq r_{i}
$$

for any $i$. We want to modify $X$ by a sequence of blowups so as to decrease $\operatorname{codim}_{x_{i}} X^{H_{i}}$ to $r_{i}$ for each $i$. (Of course, after each blowup $\widetilde{X} \longrightarrow X$ we replace $X$ by $\widetilde{X}$ and $x_{i}$ by $\widetilde{x_{i}}$, as in Lemma 9.4.) We claim that we may do this for one $i$ at a time; in other words, we may assume $s=1$. Indeed, suppose we have reduced to the case where $\operatorname{codim}_{x_{1}}\left(X^{H_{1}}\right)=r_{1}$. If we now perform a further blowup $\widetilde{X} \longrightarrow X$ and choose $\widetilde{x_{1}}$ above $x_{1}$ as in Lemma 9.4, then Lemma 9.4 and (9.3) tell us that $\operatorname{codim}_{\widetilde{x_{1}}}\left(\widetilde{X}^{H_{1}}\right)=r_{1}$. Thus we are free to perform another sequence of blowups that would give us the desired equality for $i=2$, then $i=3$, etc.

We will thus assume $s=1$ and set $x=x_{1}, H=H_{1}, r=r_{1}$.

After performing a sequence of blowups given by Lemma 9.2, we may assume that there exist $G$-invariant divisors $D_{1}, \ldots, D_{c}$ such that $X^{H}=$ $D_{1} \cap \cdots \cap D_{c}$ in a neighborhood of $x, c=\operatorname{codim}_{x} X^{H}$ and $D_{1}, \ldots, D_{c}$ intersect at $x$ transversely.

Note that $T_{x}(X) / T_{x}\left(X_{i}^{H}\right) \cong \bigoplus_{j=1}^{c} T_{x}(X) / T_{x}\left(D_{j}\right)$. Here $H$ acts on each one-dimensional space $T_{x}(X) / T_{x}\left(D_{i}\right)$ by a character $\xi_{i} \in H^{*}$ which is nontrivial by Lemma 9.1(1)(iii). In other words, the linear action of $H$ on the tangent space $T_{x}(X)$ decomposes as a direct sum of $c$ nontrivial characters $\xi_{1}, \ldots, \xi_{c}$ and $n-c=\operatorname{dim} X^{H}$ trivial characters.

Recall that by (9.3), $c \geq r$. We would like to modify $X$ by a sequence of blowups to arrive at the situation where $c=r$. In other words, if $c>r$, we want to perform a sequence of blowups that would lower the value of $c$.

With this goal in mind, we would like to know how the characters $\xi_{i}$ change after one blowup. Specifically, we will consider the blowup $\pi: \widetilde{X} \longrightarrow$ $X$ with center $Z=D_{i} \cap D_{j}$, where $1 \leq i, j \leq c, i \neq j$. Since $Z$ is of codimension 2 in $X, \pi^{-1}(x)$ is isomorphic to $\mathbb{P}^{1}$. Let $\widetilde{x}$ be the (unique) point of $\pi^{-1}(x)$ that lies in the strict transform of $D_{i}$, take $\widetilde{D}_{l}$ to be the strict transform of $D_{l}$ for $l=1, \ldots, \widehat{j}, \ldots, c$, and let $\widetilde{D}_{j}=\pi^{-1}(Z)$ be the exceptional divisor of $\pi$. Then the action of $H$ in $T_{\widetilde{x}} \widetilde{X}$ is given by the direct sum of the characters $\widetilde{\xi}_{l}=\xi_{l}$ if $l \neq i, \widetilde{\xi}_{i}=\xi_{i}{\underset{\widetilde{\xi}}{\xi_{1}}}^{-1}$, and $(\operatorname{dim} X-c)$ trivial characters. In other words, the new characters $\widetilde{\xi}_{1}, \ldots, \widetilde{\xi}_{c} \in H^{*}$ are obtained 
from the old characters $\xi_{1}, \ldots, \xi_{c} \in H^{*}$ by an elementary operation, as in Lemma 9.6. (Note that our group $H^{*}$ is written multiplicatively, whereas the group $A$ in Lemma 9.6 is written additively.)

Now Lemma 9.6 tells us that there is a sequence of of elementary operations which transforms $\left(\xi_{1}, \ldots, \xi_{c}\right)$ to $\left(\lambda_{1}, \ldots, \lambda_{r}, 1_{H^{*}}, \ldots, 1_{H^{*}}\right)$ for some $\lambda_{1}, \ldots, \lambda_{r} \in H^{*}$. Recall that initially our characters $\xi_{i}$ are all nontrivial. We want to follow the above sequence of elementary operations until we create the first trivial character. Each one of these operations is given by a blowup of a codimension 2 subvariety, as described above. When the first trivial character appears, $\operatorname{dim}\left(X^{H}\right)$ goes up by one. At that point, we reduce $c$ by 1 and repeat the above procedure, until $c$ becomes equal to $r$.

\section{A weak equivariant Bertini theorem.}

Theorem 9.7. Let $G$ be a finite group, $X$ a primitive smooth projective $G$ variety, $x_{1}, \ldots, x_{s}$ points of $X$ with stabilizers $H_{i}=\operatorname{Stab}\left(x_{i}\right)$ for $i=1, \ldots, s$. Suppose that each $x_{i}$ is not an isolated point of $X^{H_{i}}$ and $\operatorname{dim}(X) \geq 2$. Then:

(a) There exists a smooth closed $G$-invariant primitive hypersurface $W \subset$ $X$ passing through $x_{1}, \ldots, x_{s}$.

(b) Moreover, if $X$ is a generically free $G$-variety then we can choose $W$ so that it is also generically free.

We shall need the following variant of Bertini's theorem; for lack of a reference we will supply a proof.

Lemma 9.8. Let $x_{1}, \ldots, x_{s}$ be points in $\mathbb{P}^{n}$ and let $V_{d}$ be the space of homogeneous polynomials of degree $d \geq s+1$ in $\mathbb{P}^{n}$ that vanish at $x_{1}, \ldots, x_{s}$.

(a) Suppose $Y$ is a locally closed subvariety of $\mathbb{P}^{n}, y$ is a smooth point of $Y$ and $V_{d, y}=\left\{P \in V_{d}|P|_{Y}\right.$ has zero of order $>1$ at $\left.y\right\}$. Then the codimension of $V_{d, y}$ in $V_{d}$ is given by

$$
\operatorname{codim}\left(V_{d, y}\right)= \begin{cases}\operatorname{dim}_{y}(Y)+1 & \text { if } y \notin\left\{x_{1}, \ldots, x_{s}\right\}, \\ \operatorname{dim}_{y}(Y) & \text { if } y \in\left\{x_{1}, \ldots, x_{s}\right\} .\end{cases}
$$

(b) Suppose $Y_{1}, \ldots, Y_{l}$ are smooth locally closed subvarieties of $\mathbb{P}^{n}$ such that

$$
x_{j} \text { is not an isolated point of } Y_{i} \text { for any } i, j .
$$

Then a generic hypersurface of degree $d \geq s+1$ in $\mathbb{P}^{n}$ that passes through $x_{1}, \ldots, x_{s}$, is transverse to $Y_{1}, \ldots, Y_{l}$.

Note that assumption (9.4) in part (b) is necessary, since otherwise no hypersurface passing through $x_{j}$ can be transverse to $Y_{i}$. (By definition, a hypersurface $W$ is transverse to a one-point set $\left\{x_{j}\right\}$ iff $W$ does not pass through $x_{j}$.) 
Proof. (a) Choose an affine subset $\mathbb{A}^{n}=\operatorname{Spec} k\left[z_{1}, \ldots, z_{n}\right] \subset \mathbb{P}^{n}$ that contains $x_{1}, \ldots, x_{s}$ and $y$; then $V_{d}$ may be identified with the space of all polynomials in $z=\left(z_{1}, \ldots, z_{n}\right)$ of degree $\leq d$ (not necessarily homogeneous) that vanish at the points $x_{1}, \ldots, x_{s}$.

Consider the linear map $\phi_{y}: V_{d} \longrightarrow \mathcal{O}_{y} / \mathfrak{m}_{y}^{2}$, where $\mathcal{O}_{y} / \mathfrak{m}_{y}^{2}$ is the space of 1-jets of regular functions on $\mathbb{A}^{n}$ at $y ; \phi_{y}$ sends each polynomial $P \in V_{d}$ into its 1-jet at $y$ (cf. [Ha, Proof of Theorem 8.18]). Then $V_{d, y}=\phi_{y}^{-1}\left(N_{y}\right)$ where $N_{y}$ is the subspace of $\mathcal{O}_{y} / \mathfrak{m}_{y}^{2}$ consisting of the jets of all functions that vanish on $Y ; N_{y}$ may be identified with the conormal space to $Y$ at $y$, and hence, it is a linear subspace of $\mathcal{O}_{y} / \mathfrak{m}_{y}^{2}$ of $\operatorname{dimension} \operatorname{dim}\left(N_{y}\right)=\operatorname{codim}_{y}(Y)$.

Assume $y \notin\left\{x_{1}, \ldots, x_{s}\right\}$. Let $l(z)$ be a linear combination of $z_{1}, \ldots, z_{n}$ whose value at $y$ is different from its values at $x_{1}, \ldots, x_{s}$. Then the degree $s$ polynomial $P(z)=\left(l(z)-l\left(x_{1}\right)\right) \ldots\left(l(z)-l\left(x_{s}\right)\right)$ vanishes at $x_{1}, \ldots, x_{s}$ and does not vanish at $y$. This means that $\phi_{y}(P(z))$ and $\phi_{y}\left(z_{j} P(z)\right)$ (where $j=1, \ldots, n) \operatorname{span} \mathcal{O}_{y} / \mathfrak{m}_{y}^{2}$ as a $k$-vector space. Hence, $\phi_{y}$ is onto, and

$$
\begin{aligned}
\operatorname{codim}\left(V_{d, y}\right)=\operatorname{codim}\left(\phi_{y}^{-1}\left(N_{y}\right)\right)= & \operatorname{dim}\left(\mathcal{O}_{y} / \mathfrak{m}_{y}^{2}\right)-\operatorname{dim}\left(N_{y}\right) \\
& =n+1-\operatorname{codim}_{y}(Y)=\operatorname{dim}_{y}(Y)+1
\end{aligned}
$$

as claimed.

Now suppose $y \in\left\{x_{1}, \ldots, x_{s}\right\}$, say, $y=x_{1}$. In this case $\phi_{y}\left(V_{d}\right)$ is clearly contained in $\mathfrak{m}_{y} / \mathfrak{m}_{y}^{2}$; we will show that, in fact, equality holds. Indeed, we may assume without loss of generality that $y=x_{1}=(0, \ldots, 0) \in \mathbb{A}^{n}$. Let $l(z)$ be a linear combination of $z_{1}, \ldots, z_{n}$ such that $l\left(x_{2}\right), \ldots, l\left(x_{s}\right) \neq l\left(x_{1}\right)=$ 0 and let $Q(z)=\left(l(z)-l\left(x_{2}\right)\right) \ldots\left(l(z)-l\left(x_{s}\right)\right)$. Given any linear function $a(z)$ in $z_{1}, \ldots, z_{n}$, let $R(z)=a(z) Q(z)$. Note that $R(z)$ vanishes at $x_{1}, \ldots, x_{s}$ and has degree $s$; hence, $R(z) \in V_{d}$. On the other hand, $\phi_{y}(R)$ equals $R(z)$ modulo the terms of degree $\geq 2$ in $z_{1}, \ldots, z_{n}$, i.e., $\phi_{y}(R)=Q(0) a(z)(\bmod$ $\left.\mathfrak{m}_{y}^{2}\right)$, where $Q(0)$ is a nonzero element of $k$. This means that $\phi_{y}\left(V_{d}\right)$ contains $a(z)$, thus proving that $\phi_{y}\left(V_{d}\right)=\mathfrak{m}_{y} / \mathfrak{m}_{y}^{2}$, as desired. Now

$$
\begin{aligned}
\operatorname{codim}\left(V_{d, y}\right)=\operatorname{codim}\left(\phi_{y}^{-1}\left(N_{y}\right)\right)=\operatorname{dim}\left(\mathfrak{m}_{y} / \mathfrak{m}_{y}^{2}\right)-\operatorname{dim}\left(N_{y}\right) & \\
& =n-\operatorname{codim}_{y}(Y)=\operatorname{dim}_{y}(Y) .
\end{aligned}
$$

(b) After replacing each $Y_{i}$ by the collection of its irreducible components, we may assume each $Y_{i}$ is irreducible. Moreover, we may assume without loss of generality that $l=1$; we shall denote $Y_{1}$ by $Y$.

Let $X$ be the algebraic subvariety of $V_{d} \times Y$ given by

$$
X=\left\{(P, y)|P|_{Y} \text { has a zero of order }>1 \text { at } y\right\} .
$$

Denote the natural projections of $X$ to $V_{d}$ and $Y$ by $\pi_{1}$ and $\pi_{2}$. We want to show that $\operatorname{dim}\left(\pi_{1}(X)\right)<\operatorname{dim}\left(V_{d}\right)$. It is thus enough to prove that $\operatorname{dim}(X)<$ 
$\operatorname{dim}\left(V_{d}\right)$. Write $X=X_{1} \cup X_{2}$, where $X_{1}=\pi_{2}^{-1}\left(Y-\left\{x_{1}, \ldots, x_{s}\right\}\right)$ and $X_{2}=\pi_{2}^{-1}\left(Y \cap\left\{x_{1}, \ldots, x_{s}\right\}\right)$. It is enough to show that $\operatorname{dim}\left(X_{i}\right)<\operatorname{dim}\left(V_{d}\right)$ for $i=1,2$. The fibers of $\pi_{2}$ are precisely the sets $V_{d, y}$ we considered in part (a). Since $\operatorname{dim}\left(V_{d, y}\right)=\operatorname{dim}\left(V_{d}\right)-\operatorname{dim}(Y)-1$ for every $y \in Y-\left\{x_{1}, \ldots, x_{s}\right\}$, we conclude that $\operatorname{dim}\left(X_{1}\right) \leq \operatorname{dim}\left(V_{d}\right)-1$.

It remains to show that $\operatorname{dim}\left(X_{2}\right) \leq \operatorname{dim}\left(V_{d}\right)-1$. If $Y \cap\left\{x_{1}, \ldots, x_{s}\right\}=\emptyset$ then $X_{2}=\emptyset$, and there is nothing to prove. On the other hand, if $Y \cap$ $\left\{x_{1}, \ldots, x_{s}\right\} \neq \emptyset$ then assumption (9.4) says that $\operatorname{dim}(Y) \geq 1$. Thus by part (a),

$$
\operatorname{dim}\left(X_{2}\right) \leq \operatorname{dim}\left(V_{d}\right)-\operatorname{dim}(Y) \leq \operatorname{dim}\left(V_{d}\right)-1,
$$

as claimed.

Proof of Theorem 9.7. We begin with three simple observations. First of all, we may assume without loss of generality that the orbits $G x_{i}$ are disjoint. Indeed, if $W$ passes through $x_{i}$ then it will pass through every point of $G x_{i}$. Thus if, say, $x_{j}$ happens to lie in $G x_{i}$ then we can simply remove $x_{j}$ from our finite collection of points and proceed to construct $W$ for the smaller collection.

Secondly, part (b) is an immediate consequence of part (a). Indeed, since $G$ is a finite group, generically free $G$-varieties are precisely faithful $G$-varieties, i.e., $G$-varieties, where every nonidentity element of $G$ acts nontrivially. The set

$$
X_{0}=\{x \in X \mid \operatorname{Stab}(x)=\{1\}\}
$$

is open and dense in $X$; in order to ensure that $W$ is generically free, it is enough to construct $W$ so that $W \cap X_{0} \neq \emptyset$. This is accomplished by applying part (a) to the collection $\left\{x_{0}, x_{1}, \ldots, x_{s}\right\}$, where $x_{0} \in X_{0}$. Therefore, it is enough to prove part (a).

Thirdly, since $G$ is a finite group and $X$ is projective, there exists a (finite) geometric quotient morphism $\psi: X \longrightarrow X / / G$ with $X / / G$ projective; see Lemma 2.1. Since $X$ is a primitive $G$-variety, $X / / G$ is irreducible. (Recall that the geometric quotient $X / / G$ is a birational model for the rational quotient $X / G$ which is irreducible since $X$ is primitive.) Note that $X$ is partitioned into a union of nonintersecting smooth locally closed subsets $\widetilde{X^{H}}=\{x \in X \mid \operatorname{Stab}(x)=H\}$, where $H$ ranges over the set of subgroups of $G$. By the Luna Slice Theorem [PV, Theorem 6.1] the morphism

$$
\left.\psi\right|_{\widetilde{X^{H}}}: \widetilde{X^{H}} \longrightarrow \psi\left(\widetilde{X^{H}}\right)
$$

is étale, and hence, the sets $\psi\left(\widetilde{X^{H}}\right)$ are also smooth. (Note that Luna's theorem can be applied to the $G$-action in a neighborhood of any point of $X$ by Lemma 2.1(a).) Two subvarieties $\psi\left(\widetilde{X^{H}}\right)$ and $\psi\left(\widetilde{X^{H^{\prime}}}\right)$ coincide if the subgroups $H$ and $H^{\prime}$ are conjugate, and are disjoint otherwise. In other 
words, $X / / G$ is partitioned into a union of nonintersecting smooth locally closed subsets $\psi\left(\widetilde{X^{H}}\right)$, one for each conjugacy class of subgroups in $G$. Every $y \in X / / G$ lies in $\psi\left(\widehat{X^{H}}\right)$, where $H=\operatorname{Stab}(x)$ and $x \in \psi^{-1}(y)$ (the conjugacy class of $H$ does not depend on the choice of $x$ in $\left.\psi^{-1}(y)\right)$.

We are now ready to proceed with the proof of part (a). Since $X / / G$ is projective, we can embed it in $\mathbb{P}^{N}$ for some $N$. Let $U$ be a generically chosen hypersurface of degree $s+1$ in $\mathbb{P}^{N}$ which passes through $\psi\left(x_{1}\right), \ldots, \psi\left(x_{s}\right)$, and let $\bar{W}=X / / G \cap U$. We claim that

$$
W=\pi^{-1}(\bar{W}) \subset X
$$

satisfies the conditions of part (a). By our construction $W$ is a closed $G$ invariant hypersurface in $X$ passing through $x_{1}, \ldots, x_{s}$; thus we only need to show that $W$ is smooth and primitive.

Since each $x_{i}$ lies in $X^{H_{i}}$ and is not its isolated point, each $\psi\left(x_{i}\right)$ lies in $\psi\left(\widetilde{X^{H}}\right)$ and is not its isolated point; it follows that $\psi\left(x_{i}\right)$ is not an isolated point of any $\psi\left(\widetilde{X^{H}}\right)$. By Lemma $9.8(\mathrm{~b}), U$ intersects every subvariety $\psi\left(\widetilde{X^{H}}\right)$ transversely.

Let $x \in W$ and $H=\operatorname{Stab}(x)$; then $x \in \widetilde{X^{H}}$ and $\psi(x) \in \psi\left(\widetilde{X^{H}}\right)$. Let $f$ be a local equation of $U$ at $\psi(x)$; then $\psi^{*}(f)$ is a local equation of $W$ in $X$ at $x$. Since $U$ is transverse to $\psi\left(\widetilde{X^{H}}\right)$, the restriction $\left.f\right|_{\psi\left(\widetilde{X^{H}}\right)}$ is nondegenerate at $\psi(x)$. As the morphism (9.5) is étale, $\psi^{*}\left(\left.f\right|_{\psi\left(\widetilde{X^{H}}\right)}\right)=\left.\psi^{*}(f)\right|_{\widetilde{X^{H}}}$ is a nondegenerate function on $\widetilde{X^{H}}$ at $x$. Hence, $\psi^{*}(f)$ is nondegenerate at $x$; in other words, $W$ is smooth at $x$.

It remains to prove that $W$ is primitive or, equivalently, $\bar{W}$ is irreducible. By [Ha, Corollary III.7.9], $\bar{W}=X / / G \cap U$ is connected. On the other hand, since $W$ is smooth, $\bar{W}=W / / G$ is normal. We conclude that $\bar{W}$ is irreducible, as claimed.

Proof of Proposition 8.6. If $r=0$ then $H_{1}=\cdots=H_{s}=\{1\}$, and we can take $Y$ to be a set of $|G|$ points with a transitive $G$-action and $y_{1}, \ldots, y_{s}$ to be any $s$ points in $Y$ (not necessarily distinct). From now on we shall assume $r \geq 1$.

Let $V$ be a generically free linear representation of $G$. By Proposition 2.2 and Theorem 2.5, there exists a smooth projective $G$-variety $X$ such that $X \simeq V$ (as $G$-varieties) and $\operatorname{Stab}(x)$ is commutative for any $x \in X$. (Note that a Levi-commutative finite group is commutative.) Every $H_{i}$ has a smooth fixed point in $V$, namely the origin. Applying the Going Down Theorem 2.6 to the birational isomorphism $V \stackrel{\sim}{\simeq} \underset{-\rightarrow}{\rightarrow} X$, we conclude that $X^{H_{i}} \neq \emptyset$ for every $i$. The resulting smooth projective irreducible generically free $G$-variety $X$ is the starting point for our construction.

After birationally modifying $X$ by a sequence of blowups with smooth $G$-equivariant centers, we may assume that there are points $x_{1}, \ldots, x_{s} \in X$ 
such that each $x_{i}$ is fixed by $H_{i}$ and the codimension of $X^{H_{i}}$ at $x_{i}$ is $r_{i}$; see Theorem 9.3.

If $\operatorname{dim} X>r=\max _{i} r_{i}$ then $\operatorname{dim} X>r_{i}=\operatorname{codim}_{x_{i}} X^{H_{i}}$ and hence, $x_{i}$ is not an isolated fixed point of $H_{i}$ for each $i$. In addition, $\operatorname{dim} X>r \geq 1$ implies $\operatorname{dim} X \geq 2$. Then Theorem 9.7 (b) yields a smooth closed generically free $G$-invariant primitive hypersurface $W$ in $X$ passing through $x_{1}, \ldots, x_{s}$. Replacing $X$ by this hypersurface reduces $\operatorname{dim} X$ by one. Applying this procedure $\operatorname{dim} X-r$ times, we obtain a smooth $G$-invariant primitive subvariety $Y$ of dimension $r$ passing through $x_{1}, \ldots, x_{s}$, and hence, having points fixed by $H_{1}, \ldots, H_{s}$. This completes the proof of Proposition 8.6 and thus of Theorem 1.4.

Remark 9.9. A closer examination of the proof of Proposition 8.6 shows that the $G$-variety $Y$ can, in fact, be constructed over $\mathbb{Q}$. Thus the division algebra $D$ is Theorem 1.4 can be assumed to be defined over $\mathbb{Q}$. This means that there exists a finitely generated field extension $F / \mathbb{Q}$ and a division algebra $D_{0}$ with center $F$ such that $\operatorname{trdeg}_{\mathbb{Q}}(F)=6$ and $D=D_{0} \otimes_{F} K$.

Remark 9.10. Our argument can be modified to prove the following stronger form of Theorem 1.4: For any integer $e \geq 0$ there exists a division algebra $D$ with center $K$ such that

(a) $K$ is a finitely generated extension of $k$ of transcendence degree $6+2 e$ and

(b) any extension of $D$ of degree $s$ is not a crossed product, provided that $p^{e+1} \nmid s$.

\section{References}

[Ad] J.F. Adams, 2-tori in E8, Math. Ann., 287 (1987), 29-39, MR 89b:22010, Zbl 623.22011 .

$[\mathrm{BM}] \quad$ E. Bierstone and P.D. Milman, Canonical desingularization in characteristic zero by blowing up the maximum strata of a local invariant, Invent. Math., 128(2) (1997), 207-302, MR 98e:14010, Zbl 896.14006.

[Bor1] A. Borel, Sous groupes commutatifs et torsion des groupes de Lie compacts connexes, Tôhoku Math. J., 13(2) (1961), 216-240, MR 26 \#5094, Zbl 109.26101.

[Bor2] - Linear Algebraic Groups, 2nd edition, Springer-Verlag, 1991, MR 92d:20001, Zbl 726.20030.

[BS] A. Borel and J.-P. Serre, Sur certains sous groupes des groupes de Lie compacts, Comment. Math. Helv., 27 (1953), 128-139, MR 14,948d.

[Br] E. Brussel, Noncrossed products and nonabelian crossed products over $\mathbb{Q}(t)$ and $\mathbb{Q}((t))$, Amer. J. Math., 117(2) (1995), 377-393, MR 96a:16014, Zbl 822.16013.

[BR] J. Buhler and Z. Reichstein, On the essential dimension of a finite group, Compositio Math., 106 (1997), 159-179, MR 98e:12004, Zbl 905.12003.

[DC] J.A. Dieudonne and J.B. Carrell, Invariant Theory, Old and New, Academic Press, New York-Longon, 1971, MR 43 \#4828, Zbl 258.14011. 
[Ga] L.Yu. Galitskii, On the existence of Galois sections, in 'Lie Groups, their Discrete Subgroups and Invariant Theory', Advances in Soviet Math., 8 (1992), Amer. Math. Soc., Providence, RI, 65-68, MR 93d:14068.

[Gri] R.L. Griess, Jr., Elementary abelian p-subgroups of algebraic groups, Geom. Dedicata, 39 (1991), 253-305, MR 92i:20047, Zbl 733.20023.

[Gro] A. Grothendieck, La torsion homologique et les sections rationnelles, Exposé 5, Séminaire C. Chevalley, Anneaux de Chow et applications, 2nd année, IHP, 1958.

[Ha] R. Hartshorne, Algebraic Geometry, Springer, 1977, MR 57 \#3116, Zbl 367.14001.

[I] A.V. Iltyakov, On rational invariants of the group $E_{6}$, Proc. Amer. Math. Soc., 124(12) (1996), 3637-3640, MR 97b:13008, Zbl 860.20033.

[JW] B. Jacob and A.R. Wadsworth, A new construction of noncrossed product algebras, Trans. Amer. Math. Soc., 293(2) (1996), 693-721, MR 87g:16027.

[Ka] T. Kambayashi, Projective representations of algebraic groups of transformations, Amer. J. Math., 88 (1966), 199-205, MR 34 \#5826, Zbl 141.18303.

[Ko] V.E. Kordonsky, On the essential dimension and Serre's Conjecture II for exceptional groups, preprint (in Russian).

[KMRT] M.-A. Knus, A. Merkurjev, M. Rost and J.-P. Tignol, The Book of Involutions, AMS Colloquium Publications, 44 (1999), MR 2000a:16031.

[L] S. Lang, Algebra, Addison-Wesley, 1965, MR 33 \#5416, Zbl 193.34701.

[OV] A.L. Onishchik and E.B. Vinberg, Lie Groups and Algebraic Groups, SpringerVerlag, 1990, MR 91g:22001, Zbl 722.22004.

[Pf] A. Pfister, Quadratic Forms with Applications to Algebraic Geometry and Topology, London Math. Soc. Lect. Notes Ser. 217, Cambridge University Press, 1996, MR 97c:11046.

[Po] V.L. Popov, Sections in Invariant Theory, Proceedings of the Sophus Lie Memorial Conference, Scandinavian University Press, 1994, 315-361, MR 98d:14058.

[PV] V.L. Popov and E.B. Vinberg, Invariant Theory, in 'Encyclopedia of Math. Sciences', 55, Algebraic Geometry IV, edited by A.N. Parshin and I.R. Shafarevich, Springer-Verlag, 1994.

[Pr] C. Procesi, The invariant theory of $n \times n$-matrices, Advances in Math., 19 (1976), 306-381, MR 54 \#7512, Zbl 331.15021.

[Re] Z. Reichstein, On the notion of essential dimension for algebraic groups, Transformation Groups, 5(3) (2000), 265-304, CMP 1780933.

[RY] Z. Reichstein and B. Youssin, Essential dimensions of algebraic groups and a resolution theorem for $G$-varieties, with an appendix by J. Kollár and E. Szabó, Canadian J. Math., 52(5) (2000), 1018-1056, CMP 1782331.

[Ro1] M. Rosenlicht, Some basic theorems on algebraic groups, Amer. J. Math., 78 (1956), 401-443, MR 18,514a, Zbl 073.37601.

[Ro2] A remark on quotient spaces, Anais da Academia Brasileira de Ciências, 35 (1963), 487-489, MR 30 \#2009, Zbl 123.13804.

[R] L.H. Rowen, Polynomial Identities in Ring Theory, Academic Press, 1980, MR 82a:16021, Zbl 461.16001.

[RS] L.H. Rowen and D.J. Saltman, Prime to $p$ extensions of division algebras, Israel J. Math., 78 (1992), 197-207, MR 93k:12006, Zbl 795.16012. 
[Sa] D. J. Saltman, Lectures on Division Algebras, CBMS Regional Conferences Series in Mathematics, 94, Amer. Math. Soc., Providence, 1999, MR 2000f:16023, Zbl 934.16013.

[Se1] J.-P. Serre, Espaces fibrés algébriques, Exposé 1, Séminaire C. Chevalley, Anneaux de Chow et applications, 2nd année, IHP, 1958.

[Se2] _ Local Fields, Springer-Verlag, 1979, MR 82e:12016, Zbl 423.12016.

[Se3] _ Cohomologie galoisienne: Progrès et problèmes, in 'Séminaire Bourbaki', Volume 1993/94, Exposés 775-789, Astérisque, 227 (1995), 229-257, MR 97d:11063, Zbl 837.12003.

[Se4] _ Galois Cohomology, Springer, 1997, MR 98g:12007, Zbl 902.12004.

[Se5] _ Sous-groupes finis des groupes de Lie, Séminaire Bourbaki, 1998-99, 864, Juin 1999, CMP 1772682.

[SS] T.A. Springer and R. Steinberg, Conjugacy classes, in 'Seminar on algebraic groups and related Finite Groups', Lect. Notes in Math., 131 (1970), 281-312, Zbl 249.20024

[St] R. Steinberg, Torsion in reductive groups, Advances in Math., 15 (1975), 63-92, MR 50 \#7369, Zbl 312.20026.

[TA1] J.-P. Tignol and S.A. Amitsur, Kummer subfields of Maltsev-Neumann division algebras, Israel J. Math., 50(1-2) (1985), 114-144, MR 86m:16007, Zbl 564.16015.

[TA2] , Symplectic modules, Israel J. Math., 54(3) (1986), 266-290, MR 87m:16042, Zbl 599.20086.

[T1] J. Tits, Résumé de cours, Annuaire du College de France, (1991-92), 115-133.

[T2] _ Sur les degrés des extensions de corps déployant les groupes algébriques simples, C. R. Acad. Sci. Paris, t. 315, Série I (1992), 1131-1138, MR 93m:20059.

[V] O.E. Villamayor U., Patching local uniformizations, Ann. Scient. Éc. Norm. Sup., 4e série, 25 (1992), 629-677, MR 93m:14012, Zbl 782.14009.

Received September 16, 1999 and revised July 6, 2000. Z. Reichstein was partially supported by NSF grant DMS-9801675.

Department of Mathematics

Oregon State University

Corvallis OR 97331

E-mail address: zinovy@math.orst.edu

Department of Mathematics and Computer Science

UNIVERSITY OF THE NEGEV

Be'er SHEva'

ISRAEL

HASHOFAR $26 / 3$

MA'Ale AdUmim

ISRAEL

E-mail address: youssin@math.bgu.ac.il 